\title{
A study of the reaction $e^{+} e^{-} \rightarrow \mu^{+} \mu^{-} \gamma_{\mathrm{ISR}}$ at LEP and search for new physics at annihilation energies near $80 \mathrm{GeV}$
}

DELPHI Collaboration

\begin{abstract}
A study of the channel $e^{+} e^{-} \rightarrow \mu^{+} \mu^{-} \gamma_{\text {ISR }}$, where $\gamma_{\text {ISR }}$ is an initial state radiation photon, is presented using data collected by the DELPHI experiment from 1991 to 1994. The total cross-section at effective annihilation energies $\left(\sqrt{s^{\prime}}\right)$ below the $Z^{0}$ peak is obtained by using the events with relatively hard initial state radiative photon(s) $\left(E_{\gamma}>1 \mathrm{GeV}\right)$. The differential cross-section as a function of the muon polar production angle is also determined in order to extract the forward-backward asymmetries for the reaction $e^{+} e^{-} \rightarrow \mu^{+} \mu^{-}$ at energies $\sqrt{s^{\prime}}$ between 20 and $87 \mathrm{GeV}$. The ratio of the helicity cross-sections $\frac{\sigma_{L L}+\sigma_{R R}}{\sigma_{R L}+\sigma_{L R}}$, where the two subscripts stand for the helicities of the incoming $e^{-}$ and outgoing $\mu^{-}$respectively, is extracted from the differential cross-sections in order to test the Standard Model and to look for new physics near $80 \mathrm{GeV}$. No deviations from the Standard Model were found.
\end{abstract}


P.Abreu $^{21}$, W.Adam ${ }^{49}$, T.Adye ${ }^{36}$, I.Ajinenko ${ }^{41}$, G.D.Alekseev ${ }^{16}$, R.Alemany ${ }^{48}$, P.P.Allport ${ }^{22}$, S.Almehed $^{24}$, U.Amaldi $^{9}$, S.Amato ${ }^{46}$, P.Andersson ${ }^{43}$, A.Andreazza ${ }^{9}$, P.Antilogus ${ }^{9}$, W-D.Apel ${ }^{17}$, B.Asman ${ }^{43}$, J-E.Augustin ${ }^{25}$, A.Augustinus $^{30}$, P.Baillon ${ }^{9}$, P.Bambade ${ }^{19}$, F.Barao $^{21}$, M.Barbi ${ }^{46}$, G.Barbiellini ${ }^{45}$, D.Y.Bardin ${ }^{16}$, G.Barker ${ }^{9}$,

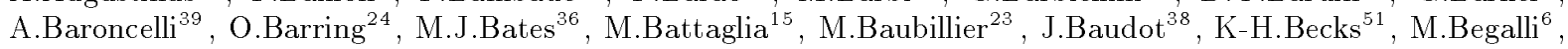
P.Beilliere $^{8}$, Yu.Belokopytov ${ }^{9,52}$, K.Belous ${ }^{41}$, A.C.Benvenuti ${ }^{5}$, M.Berggren ${ }^{46}$, D.Bertini ${ }^{25}$, D.Bertrand ${ }^{2}$, M.Besancon $^{38}$, F.Bianchi ${ }^{44}$, M.Bigi ${ }^{44}$, M.S.Bilenky ${ }^{16}$, P.Billoir ${ }^{23}$, M-A.Bizouard ${ }^{19}$, D.Bloch ${ }^{10}$, M.Blume ${ }^{51}$, M.Bonesini $^{27}$, W.Bonivento ${ }^{27}$, P.S.L.Booth ${ }^{22}$, A.W.Borgland ${ }^{4}$, G.Borisov ${ }^{38,41}$, C.Bosio ${ }^{39}$, O.Botner $^{47}$, E.Boudinov $^{30}$, B.Bouquet ${ }^{19}$, C.Bourdarios ${ }^{19}$, T.J.V.Bowcock ${ }^{22}$, I.Bozovic ${ }^{11}$, M.Bozzo ${ }^{13}$, P.Branchini ${ }^{39}$, K.D.Brand ${ }^{35}$, T.Brenke ${ }^{51}$, R.A.Brenner ${ }^{47}$, C.Bricman ${ }^{2}$, R.C.A.Brown ${ }^{9}$, P.Bruckman ${ }^{18}$, J-M.Brunet ${ }^{8}$, L.Bugge ${ }^{32}$, T.Buran $^{32}$, T.Burgsmueller ${ }^{51}$, P.Buschmann ${ }^{51}$, S.Cabrera ${ }^{48}$, M.Caccia ${ }^{27}$, M.Calvi ${ }^{27}$, A.J.Camacho Rozas ${ }^{40}$, T.Camporesi $^{9}$, V.Canale ${ }^{37}$, M.Canepa ${ }^{13}$, F.Cao $^{2}$,F.Carena ${ }^{9}$, L.Carroll ${ }^{22}$, C.Caso ${ }^{13}$, M.V.Castillo Gimenez ${ }^{48}$, A.Cattai $^{9}$, F.R.Cavallo ${ }^{5}$, V.Chabaud ${ }^{9}$, Ph.Charpentier ${ }^{9}$, L.Chaussard ${ }^{25}$, P.Chechia ${ }^{35}$, G.A.Chelkov ${ }^{16}$, M.Chen $^{2}$, R.Chierici ${ }^{44}$, P.Chliapnikov ${ }^{41}$, P.Chochula ${ }^{7}$, V.Chorowicz ${ }^{25}$, J.Chudoba ${ }^{29}$, V.Cindro ${ }^{42}$, P.Collins ${ }^{9}$, R.Contri ${ }^{13}$, E.Cortina ${ }^{48}$, G.Cosme ${ }^{19}$, F.Cossutti ${ }^{45}$, J-H.Cowell ${ }^{22}$, H.B.Crawley ${ }^{1}$, D.Crennell ${ }^{36}$, G.Crosetti $^{13}$, J.Cuevas Maestro $^{33}$, S.Czellar ${ }^{15}$, J.Dahm ${ }^{51}$, B.Dalmagne ${ }^{19}$, M.Dam ${ }^{28}$, G.Damgaard ${ }^{28}$, P.D.Dauncey ${ }^{36}$, M.Davenport ${ }^{9}$, W.Da Silva ${ }^{23}$, A.Deghorain ${ }^{2}$, G.Della Ricca ${ }^{45}$, P.Delpierre ${ }^{26}$, N.Demaria ${ }^{34}$, A.De Angelis ${ }^{9}$, W.De Boer ${ }^{17}$, S.De Brabandere ${ }^{2}$, C.De Clercq ${ }^{2}$, C.De La Vaissiere ${ }^{23}$, B.De Lotto ${ }^{45}$, A.De Min ${ }^{35}$, L.De Paula ${ }^{46}$, H.Dijkstra ${ }^{9}$, L.Di Ciaccio ${ }^{37}$, A.Di Diodato ${ }^{37}$, A.Djannati ${ }^{8}$, J.Dolbeau ${ }^{8}$, K.Doroba ${ }^{50}$, M.Dracos ${ }^{10}$, J.Drees ${ }^{51}$, K.-A.Drees ${ }^{51}$, M.Dris ${ }^{31}$, J-D.Durand ${ }^{25,9}$, D.Edsall ${ }^{1}$, R.Ehret ${ }^{17}$, G.Eigen ${ }^{4}$, T.Ekelof ${ }^{47}$, G.Ekspong ${ }^{43}$, M.Elsing ${ }^{9}$, J-P.Engel $^{10}$, B.Erzen ${ }^{42}$, M.Espirito Santo ${ }^{21}$, E.Falk ${ }^{24}$, G.Fanourakis ${ }^{11}$, D.Fassouliotis ${ }^{45}$, M.Feindt ${ }^{9}$, A.Fenyuk ${ }^{41}$, P.Ferrari $^{27}$, A.Ferrer ${ }^{48}$, S.Fichet ${ }^{23}$, T.A.Filippas ${ }^{31}$, A.Firestone ${ }^{1}$, P.-A.Fischer ${ }^{10}$, H.Foeth ${ }^{9}$, E.Fokitis ${ }^{31}$, F.Fontanelli ${ }^{13}$, F.Formenti ${ }^{9}$, B.Franek ${ }^{36}$, A.G.Frodesen ${ }^{4}$, R.Fruhwirth ${ }^{49}$, F.Fulda-Quenzer ${ }^{19}$, J.Fuster ${ }^{48}$, A.Galloni $^{22}$, D.Gamba ${ }^{44}$, M.Gandelman ${ }^{46}$, C.Garcia $^{48}$, J.Garcia ${ }^{40}$, C.Gaspar ${ }^{9}$, U.Gasparini ${ }^{35}$, Ph.Gavillet ${ }^{9}$, E.N.Gazis ${ }^{31}$, D.Gele ${ }^{10}$, J-P.Gerber ${ }^{10}$, L.Gerdyukov ${ }^{41}$, R.Gokieli ${ }^{50}$, B.Golob ${ }^{42}$, P.Goncalves ${ }^{21}$, G.Gopal $^{36}$, L.Gorn $^{1}$, M.Gorski ${ }^{50}$, Yu.Gouz ${ }^{4,52}$, V.Gracco ${ }^{13}$, E.Graziani ${ }^{39}$, C.Green ${ }^{22}$, A.Grefrath ${ }^{51}$, P.Gris ${ }^{38}$, G.Grosdidier $^{19}$, K.Grzelak ${ }^{50}$, S.Gumenyuk ${ }^{41}$, M.Gunther ${ }^{47}$, J.Guy ${ }^{36}$, F.Hahn ${ }^{9}$, S.Hahn ${ }^{51}$, Z.Hajduk ${ }^{18}$, A.Hallgren $^{47}$, K.Hamacher ${ }^{51}$, F.J.Harris ${ }^{34}$, V.Hedberg ${ }^{24}$, J.J.Hernandez ${ }^{48}$, P.Herquet ${ }^{2}$, H.Herr ${ }^{9}$, T.L.Hessing ${ }^{34}$, J.-M.Heuser $^{51}$, E.Higon ${ }^{48}$, H.J.Hilke ${ }^{9}, \quad$ S-O.Holmgren ${ }^{43}$, P.J.Holt ${ }^{34}$, D.Holthuizen ${ }^{30}$, S.Hoorelbeke ${ }^{2}$, M.Houlden $^{22}$, J.Hrubec ${ }^{49}$, K.Huet ${ }^{2}$, K.Hultqvist ${ }^{43}$, J.N.Jackson ${ }^{22}$, R.Jacobsson ${ }^{43}$, P.Jalocha ${ }^{9}$, R.Janik ${ }^{7}$, Ch.Jarlskog $^{24}$, G.Jarlskog ${ }^{24}$, P.Jarry ${ }^{38}$, B.Jean-Marie ${ }^{19}$, E.K.Johansson ${ }^{43}$, L.Jonsson ${ }^{24}$, P.Jonsson ${ }^{24}$, C.Joram ${ }^{9}$, P.Juillot ${ }^{10}$, M.Kaiser ${ }^{17}$, F.Kapusta ${ }^{23}$, K.Karafasoulis ${ }^{11}$, M.Karlsson ${ }^{43}$, S.Katsanevas ${ }^{25}$, E.C.Katsoufis ${ }^{31}$, R.Keranen $^{4}$, Yu.Khokhlov ${ }^{41}$, B.A.Khomenko ${ }^{16}$, N.N.Khovanski ${ }^{16}$, B.King ${ }^{22}$, N.J.Kjaer ${ }^{30}$, O.Klapp ${ }^{51}$, H.Klein ${ }^{9}$, P.Kluit $^{30}$, D.Knoblauch ${ }^{17}$, P.Kokkinias ${ }^{11}$, M.Koratzinos ${ }^{9}$, K.Korcyl ${ }^{18}$, V.Kostioukhine ${ }^{41}$, C.Kourkoumelis ${ }^{3}$, O.Kouznetsov ${ }^{13,16}$, M.Krammer ${ }^{49}$, C.Kreuter ${ }^{9}$, I.Kronkvist ${ }^{24}$, Z.Krumstein ${ }^{16}$, W.Krupinski ${ }^{18}$, P.Kubinec ${ }^{7}$, W.Kucewicz ${ }^{18}$, K.Kurvinen ${ }^{15}$, C.Lacasta ${ }^{9}$, I.Laktineh ${ }^{25}$, J.W.Lamsa ${ }^{1}$, L.Lanceri ${ }^{45}$, D.W.Lane ${ }^{1}$, P.Langefeld ${ }^{51}$, J-P.Laugier $^{38}$, R.Lauhakangas ${ }^{15}$, G.Leder ${ }^{49}$, F.Ledroit ${ }^{14}$, V.Lefebure ${ }^{2}$, C.K.Legan ${ }^{1}$, A.Leisos ${ }^{11}$, R.Leitner ${ }^{29}$, J.Lemonne $^{2}$, G.Lenzen ${ }^{51}$, V.Lepeltier ${ }^{19}$, T.Lesiak ${ }^{18}$, J.Libby ${ }^{34}$, D.Liko ${ }^{9}$, A.Lipniacka ${ }^{43}$, I.Lippi $^{35}$, B.Loerstad $^{24}$, J.G.Loken ${ }^{34}$, J.M.Lopez ${ }^{40}$, D.Loukas ${ }^{11}$, P.Lutz ${ }^{38}$, L.Lyons ${ }^{34}$, G.Maehlum ${ }^{17}$, J.R.Mahon ${ }^{6}$, A.Maio ${ }^{21}$, T.G.M.Malmgren ${ }^{43}$, V.Malychev ${ }^{16}$, F.Mandl ${ }^{49}$, J.Marco ${ }^{40}$, R.Marco ${ }^{40}$, B.Marechal ${ }^{46}$, M.Margoni ${ }^{35}$, J-C.Marin $^{9}$, C.Mariotti ${ }^{9}$, A.Markou ${ }^{11}$, C.Martinez-Rivero ${ }^{33}$, F.Martinez-Vidal ${ }^{48}$, S.Marti i Garcia ${ }^{22}$, J.Masik $^{29}$, F.Matorras $^{40}$, C.Matteuzzi ${ }^{27}$, G.Matthiae ${ }^{37}$, M.Mazzucato ${ }^{35}$, M.Mc Cubbin ${ }^{22}$, R.Mc Kay ${ }^{1}$, R.Mc Nulty ${ }^{9}$, J.Medbo $^{47}$, C.Meroni ${ }^{27}$, S.Meyer ${ }^{17}$, W.T.Meyer ${ }^{1}$, M.Michelotto ${ }^{35}$, E.Migliore ${ }^{44}$, L.Mirabito ${ }^{25}$, W.A.Mitaroff ${ }^{49}$, U.Mjoernmark $^{24}$, T.Moa ${ }^{43}$, R.Moeller ${ }^{28}$, K.Moenig ${ }^{9}$, M.R.Monge ${ }^{13}$, P.Morettini ${ }^{13}$, H.Mueller ${ }^{17}$, K.Muenich ${ }^{51}$, M.Mulders $^{30}$, L.M.Mundim ${ }^{6}$, W.J.Murray ${ }^{36}$, B.Muryn ${ }^{14,18}$, G.Myatt ${ }^{34}$, T.Myklebust ${ }^{32}$, F.Naraghi ${ }^{14}$, F.L.Navarria ${ }^{5}$, S.Navas ${ }^{48}$, K.Nawrocki ${ }^{50}$, P.Negri ${ }^{27}$, S.Nemecek ${ }^{12}$, W.Neumann ${ }^{51}$, N.Neumeister ${ }^{49}$, R.Nicolaidou $^{3}$, B.S.Nielsen ${ }^{28}$, M.Nieuwenhuizen $^{30}$, V.Nikolaenko ${ }^{10}$, M.Nikolenko ${ }^{10,16}$, P.Niss ${ }^{43}$, A.Nomerotski $^{35}{ }^{\prime}$, A.Normand $^{34}$, W.Oberschulte-Beckmann ${ }^{17}$, V.Obraztsov ${ }^{41}$, A.G.Olshevski ${ }^{16}$, A.Onofre ${ }^{21}$, R.Orava ${ }^{15}$, G.Orazi $^{10}$, K.Osterberg ${ }^{15}$, A.Ouraou ${ }^{38}$, P.Paganini ${ }^{19}$, M.Paganoni ${ }^{9,27}$, R.Pain ${ }^{23}$, H.Palka ${ }^{18}$, Th.D.Papadopoulou ${ }^{31}$, K.Papageorgiou ${ }^{11}$, L.Pape ${ }^{9}$, C.Parkes ${ }^{34}$, F.Parodi ${ }^{13}$, A.Passeri ${ }^{39}$, M.Pegoraro $^{35}$, L.Peralta $^{21}$, H.Pernegger ${ }^{49}$, M.Pernicka ${ }^{49}$, A.Perrotta ${ }^{5}$, C.Petridou ${ }^{45}$, A.Petrolini ${ }^{13}$, H.T.Phillips ${ }^{36}$, G.Piana $^{13}$, F.Pierre $^{38}$, M.Pimenta ${ }^{21}$, T.Podobnik ${ }^{42}$, O.Podobrin ${ }^{9}$, M.E.Pol ${ }^{6}$, G.Polok ${ }^{18}$, P.Poropat ${ }^{45}$, V.Pozdniakov ${ }^{16}$, P.Privitera $^{37}$, N.Pukhaeva ${ }^{16}$, A.Pullia ${ }^{27}$, D.Radojicic ${ }^{34}$, S.Ragazzi ${ }^{27}$, H.Rahmani ${ }^{31}$, P.N.Ratoff ${ }^{20}$, A.L.Read ${ }^{32}$, M.Reale $^{51}$, P.Rebecchi ${ }^{19}$, N.G.Redaelli ${ }^{27}$, M.Regler ${ }^{49}$, D.Reid ${ }^{9}$, R.Reinhardt ${ }^{51}$, P.B.Renton ${ }^{34}$, L.K.Resvanis ${ }^{3}$, F.Richard ${ }^{19}$, J.Richardson ${ }^{22}$, J.Ridky ${ }^{12}$, G.Rinaudo ${ }^{44}$, O.Rohne ${ }^{32}$, A.Romero ${ }^{44}$, P.Ronchese ${ }^{35}$, L.Roos ${ }^{23}$, E.I.Rosenberg ${ }^{1}$, P.Roudeau ${ }^{19}$, T.Rovelli ${ }^{5}$, V.Ruhlmann-Kleider ${ }^{38}$, A.Ruiz ${ }^{40}$, K.Rybicki ${ }^{18}$, H.Saarikko ${ }^{15}$, Y.Sacquim $^{38}$, A.Sadovsky ${ }^{16}$, O.Sahr ${ }^{14}$, G.Sajot ${ }^{14}$, J.Salt ${ }^{48}$, M.Sannino ${ }^{13}$, H.Schneider ${ }^{17}$, U.Schwickerath ${ }^{17}$, M.A.E.Schyns ${ }^{51}$, G.Sciolla ${ }^{44}$, F.Scuri ${ }^{45}$, P.Seager ${ }^{20}$, Y.Sedykh ${ }^{16}$, A.M.Segar ${ }^{34}$, A.Seitz ${ }^{17}$, R.Sekulin ${ }^{36}$, L.Serbelloni $^{37}$, R.C.Shellard ${ }^{6}$, P.Siegrist ${ }^{9,38}$, R.Silvestre ${ }^{38}$, F.Simonetto ${ }^{35}$, A.N.Sisakian ${ }^{16}$, B.Sitar $^{7}$, T.B.Skaali $^{32}$, G.Smadja ${ }^{25}$, N.Smirnov ${ }^{41}$, O.Smimova ${ }^{24}$, G.R.Smith ${ }^{36}$, O.Solovianov ${ }^{41}$, R.Sosnowski ${ }^{50}$, D.Souza-Santos ${ }^{6}$, T.Spassov ${ }^{21}$, E.Spiriti ${ }^{39}$, P.Sponholz ${ }^{51}$, S.Squarcia ${ }^{13}$, D.Stampfer ${ }^{9}$, C.Stanescu ${ }^{39}$, S.Stanic ${ }^{42}$, S.Stapnes $^{32}$, I.Stavitski ${ }^{35}$, K.Stevenson ${ }^{34}$, A.Stocchi ${ }^{19}$, J.Strauss ${ }^{49}$, R.Strub ${ }^{10}$, B.Stugu ${ }^{4}$, M.Szczekowski $^{50}$, M.Szeptycka $^{50}$, T.Tabarelli ${ }^{27}$, J.P.Tavernet ${ }^{23}$, O.Tchikilev ${ }^{41}$, F.Tegenfeldt ${ }^{47}$, F.Terranova ${ }^{27}$, J.Thomas ${ }^{34}$, A.Tilquin ${ }^{26}$, J.Timmermans ${ }^{30}$, L.G.Tkatchev ${ }^{16}$, T.Todorov ${ }^{10}$, S.Todorova ${ }^{10}$, D.Z.Toet ${ }^{30}$, A.Tomaradze ${ }^{2}$, 
B.Tome ${ }^{21}$, A.Tonazzo ${ }^{27}$, L.Tortora ${ }^{39}$, G.Transtromer ${ }^{24}$, D.Treille ${ }^{9}$, G.Tristram ${ }^{8}$, A.Trombini ${ }^{19}$, C.Troncon ${ }^{27}$, A.Tsirou $^{9}$, M-L.Turluer ${ }^{38}$, I.A.Tyapkin ${ }^{16}$, M.Tyndel $^{36}$, S.Tzamarias ${ }^{11}$, B.Ueberschaer ${ }^{51}$, O.Ullaland ${ }^{9}$, V.Uvarov $^{41}$, G.Valenti ${ }^{5}$, E.Vallazza ${ }^{45}$, C.Vander Velde ${ }^{2}$, G.W.Van Apeldoorn ${ }^{30}, \quad$ P.Van Dam ${ }^{30}$, W.K.Van Doninck ${ }^{2}$, J.Van Eldik ${ }^{30}$, A.Van Lysebetten ${ }^{2}$, N.Vassilopoulos ${ }^{34}$, G.Vegni ${ }^{27}$, L.Ventura ${ }^{35}$, W.Venus ${ }^{36}$, F.Verbeure $^{2}$, M.Verlato ${ }^{35}$, L.S.Vertogradov ${ }^{16}$, D.Vilanova ${ }^{38}$, P.Vincent ${ }^{25}$, L.Vitale ${ }^{45}$, E.Vlasov ${ }^{41}$, A.S.Vodopyanov ${ }^{16}$, V.Vrba ${ }^{12}$, H.Wahlen ${ }^{51}$, C.Walck ${ }^{43}$, P.Weilhammer ${ }^{9}$ C.Weiser ${ }^{17}$, A.M.Wetherell ${ }^{9}$, D.Wicke $^{51}$, J.H.Wickens ${ }^{2}$, M.Wielers ${ }^{17}$, G.R.Wilkinson ${ }^{9}$, W.S.C.Williams ${ }^{34}$, M.Winter ${ }^{10}$, M.Witek ${ }^{18}$, T.Wlodek $^{19}$, J.Yi ${ }^{1}$, K.Yip ${ }^{34}$, O.Yushchenko ${ }^{41}$, F.Zach $^{25}$, A.Zaitsev ${ }^{41}$, A.Zalewska ${ }^{9}$, P.Zalewski ${ }^{50}$, D.Zavrtanik ${ }^{42}$, E.Zevgolatakos ${ }^{11}$, N.I.Zimin ${ }^{16}$, G.C.Zucchelli ${ }^{43}$, G.Zumerle $^{35}$

\footnotetext{
${ }^{1}$ Department of Physics and Astronomy, Iowa State University, Ames IA 50011-3160, USA

${ }^{2}$ Physics Department, Univ. Instelling Antwerpen, Universiteitsplein 1, B-2610 Wilrijk, Belgium and IIHE, ULB-VUB, Pleinlaan 2, B-1050 Brussels, Belgium and Faculté des Sciences, Univ. de l'Etat Mons, Av. Maistriau 19, B-7000 Mons, Belgium ${ }^{3}$ Physics Laboratory, University of Athens, Solonos Str. 104, GR-10680 Athens, Greece ${ }^{4}$ Department of Physics, University of Bergen, Allégaten 55, N-5007 Bergen, Norway ${ }^{5}$ Dipartimento di Fisica, Università di Bologna and INFN, Via Irnerio 46, I-40126 Bologna, Italy ${ }^{6}$ Centro Brasileiro de Pesquisas Físicas, rua Xavier Sigaud 150, RJ-22290 Rio de Janeiro, Brazil and Depto. de Física, Pont. Univ. Católica, C.P. 38071 RJ-22453 Rio de Janeiro, Brazil and Inst. de Física, Univ. Estadual do Rio de Janeiro, rua São Francisco Xavier 524, Rio de Janeiro, Brazil ${ }^{7}$ Comenius University, Faculty of Mathematics and Physics, Mlynska Dolina, SK-84215 Bratislava, Slovakia

${ }^{8}$ Collège de France, Lab. de Physique Corpusculaire, IN2P3-CNRS, F-75231 Paris Cedex 05, France

${ }^{9}$ CERN, CH-1211 Geneva 23, Switzerland

${ }^{10}$ Centre de Recherche Nucléaire, IN2P3 - CNRS/ULP - BP20, F-67037 Strasbourg Cedex, France

${ }^{11}$ Institute of Nuclear Physics, N.C.S.R. Demokritos, P.O. Box 60228, GR-15310 Athens, Greece

${ }^{12}$ FZU, Inst. of Physics of the C.A.S. High Energy Physics Division, Na Slovance 2, 180 40, Praha 8, Czech Republic

${ }^{13}$ Dipartimento di Fisica, Università di Genova and INFN, Via Dodecaneso 33, I-16146 Genova, Italy

${ }^{14}$ Institut des Sciences Nucléaires, IN2P3-CNRS, Université de Grenoble 1, F-38026 Grenoble Cedex, France

${ }^{15}$ Helsinki Institute of Physics, HIP, P.O. Box 9, FIN-00014 Helsinki, Finland

${ }^{16}$ Joint Institute for Nuclear Research, Dubna, Head Post Office, P.O. Box 79, 101000 Moscow, Russian Federation

${ }^{17}$ Institut für Experimentelle Kernphysik, Universität Karlsruhe, Postfach 6980, D-76128 Karlsruhe, Germany

${ }^{18}$ Institute of Nuclear Physics and University of Mining and Metalurgy, Ul. Kawiory 26a, PL-30055 Krakow, Poland

${ }^{19}$ Université de Paris-Sud, Lab. de l'Accélérateur Linéaire, IN2P3-CNRS, Bât. 200, F-91405 Orsay Cedex, France

${ }^{20}$ School of Physics and Chemistry, University of Lancaster, Lancaster LA1 4YB, UK

${ }^{21}$ LIP, IST, FCUL - Av. Elias Garcia, 14-1 ${ }^{\circ}$, P-1000 Lisboa Codex, Portugal

${ }^{22}$ Department of Physics, University of Liverpool, P.O. Box 147, Liverpool L69 3BX, UK

${ }^{23}$ LPNHE, IN2P3-CNRS, Universités Paris VI et VII, Tour 33 (RdC), 4 place Jussieu, F-75252 Paris Cedex 05, France

${ }^{24}$ Department of Physics, University of Lund, Sölvegatan 14, S-22363 Lund, Sweden

${ }^{25}$ Université Claude Bernard de Lyon, IPNL, IN2P3-CNRS, F-69622 Villeurbanne Cedex, France

${ }^{26}$ Univ. d'Aix - Marseille II - CPP, IN2P3-CNRS, F-13288 Marseille Cedex 09, France

${ }^{27}$ Dipartimento di Fisica, Università di Milano and INFN, Via Celoria 16, I-20133 Milan, Italy

${ }^{28}$ Niels Bohr Institute, Blegdamsvej 17, DK-2100 Copenhagen 0, Denmark

${ }^{29}$ NC, Nuclear Centre of MFF, Charles University, Areal MFF, V Holesovickach 2, 180 00, Praha 8, Czech Republic

${ }^{30}$ NIKHEF, Postbus 41882, NL-1009 DB Amsterdam, The Netherlands

${ }^{31}$ National Technical University, Physics Department, Zografou Campus, GR-15773 Athens, Greece

${ }^{32}$ Physics Department, University of Oslo, Blindern, N-1000 Oslo 3, Norway

${ }^{33}$ Dpto. Fisica, Univ. Oviedo, Avda. Calvo Sotelo, S/N-33007 Oviedo, Spain, (CICYT-AEN96-1681)

${ }^{34}$ Department of Physics, University of Oxford, Keble Road, Oxford OX1 3RH, UK

${ }^{35}$ Dipartimento di Fisica, Università di Padova and INFN, Via Marzolo 8, I-35131 Padua, Italy

${ }^{36}$ Rutherford Appleton Laboratory, Chilton, Didcot OX11 OQX, UK

${ }^{37}$ Dipartimento di Fisica, Università di Roma II and INFN, Tor Vergata, I-00173 Rome, Italy

${ }^{38}$ CEA, DAPNIA/Service de Physique des Particules, CE-Saclay, F-91191 Gif-sur-Yvette Cedex, France

${ }^{39}$ Istituto Superiore di Sanità, Ist. Naz. di Fisica Nucl. (INFN), Viale Regina Elena 299, I-00161 Rome, Italy

${ }^{40}$ Instituto de Fisica de Cantabria (CSIC-UC), Avda. los Castros, S/N-39006 Santander, Spain, (CICYT-AEN96-1681)

${ }^{41}$ Inst. for High Energy Physics, Serpukov P.O. Box 35, Protvino, (Moscow Region), Russian Federation

${ }^{42}$ J. Stefan Institute, Jamova 39, SI-1000 Ljubljana, Slovenia and Department of Astroparticle Physics, School of Environmental Sciences, Kostanjeviska 16a, Nova Gorica, SI-5000 Slovenia, and Department of Physics, University of Ljubljana, SI-1000 Ljubljana, Slovenia

${ }^{43}$ Fysikum, Stockholm University, Box 6730, S-113 85 Stockholm, Sweden

${ }^{44}$ Dipartimento di Fisica Sperimentale, Università di Torino and INFN, Via P. Giuria 1, I-10125 Turin, Italy

${ }^{45}$ Dipartimento di Fisica, Università di Trieste and INFN, Via A. Valerio 2, I-34127 Trieste, Italy and Istituto di Fisica, Università di Udine, I-33100 Udine, Italy

${ }^{46}$ Univ. Federal do Rio de Janeiro, C.P. 68528 Cidade Univ., Ilha do Fundão BR-21945-970 Rio de Janeiro, Brazil

${ }^{47}$ Department of Radiation Sciences, University of Uppsala, P.O. Box 535, S-751 21 Uppsala, Sweden

${ }^{48}$ IFIC, Valencia-CSIC, and D.F.A.M.N., U. de Valencia, Avda. Dr. Moliner 50, E-46100 Burjassot (Valencia), Spain

${ }^{49}$ Institut für Hochenergiephysik, Österr. Akad. d. Wissensch., Nikolsdorfergasse 18, A-1050 Vienna, Austria

${ }^{50}$ Inst. Nuclear Studies and University of Warsaw, Ul. Hoza 69, PL-00681 Warsaw, Poland

${ }^{51}$ Fachbereich Physik, University of Wuppertal, Postfach 100 127, D-42097 Wuppertal, Germany

${ }^{52}$ On leave of absence from IHEP Serpukhov
} 


\section{Introduction}

In this paper, experimental results from studies of events collected at LEP in the channel $e^{+} e^{-} \rightarrow \mu^{+} \mu^{-} \gamma_{\mathrm{ISR}}$, with $\gamma_{\mathrm{ISR}}$ being an initial state radiation photon, are used to probe the cross-sections and forward-backward asymmetries in the energy region between LEP and TRISTAN and down to PETRA energies. An analysis in terms of helicity cross-sections is also performed, and is shown to be particularly useful in a search for new physics near the effective annihilation energy $\sqrt{s^{\prime}}=80 \mathrm{GeV}$.

The investigation of the $e^{+} e^{-} \rightarrow \mu^{+} \mu^{-}$cross-section at energies below the $Z^{0}$ peak is attractive because no experiments have ever taken data at energies between 61 and $88 \mathrm{GeV}$. In addition, an indication of a deviation of the cross-section from the predictions of the Standard Model (SM) at $58 \mathrm{GeV}$ has been reported from TRISTAN [1]. Measurable deviations in the $e^{+} e^{-} \rightarrow f \bar{f}$ cross-section in this energy range can arise in several models beyond the SM, for instance those which introduce an additional $Z^{\prime}$ gauge boson.

In a previous paper [2] an analysis was presented of radiative muon events using the data collected by DELPHI up to 1992. In this paper a new analysis is presented based on the data collected between 1991 and 1994, and on an improved event selection procedure.

The paper is organised as follows. Section 2 summarises the theoretical background to the analysis. Section 3 describes briefly the DELPHI detector and presents the data samples used. Section 4 discusses the event selection procedures. Section 5 presents the calculation of the total Improved Born cross-sections. The analysis of the differential cross-sections in terms of the forward-backward asymmetry and the helicity cross-sections is presented in Section 6. Finally, Section 7 summarises.

\section{Theoretical background}

\subsection{Total cross-section}

Electromagnetic radiative corrections to the interaction $e^{+} e^{-} \rightarrow f \bar{f}$ substantially distort the Born-level cross-section at energies around the $Z^{0}$ pole $[3,4]$.

Most of the effects can be understood to arise from initial state radiation (ISR), after which the effective annihilation energy $\sqrt{s^{\prime}}$ of the $e^{+} e^{-}$system is less than the total centre-of-mass energy $\sqrt{s}$. Because the Born cross-section varies rapidly across the $Z^{0}$ pole, the resulting changes in the cross-section are large. Consequently they need to be understood very precisely in order to study the underlying electroweak physics.

The cross-section for the reaction $e^{+} e^{-} \rightarrow \mu^{+} \mu^{-}$has contributions from direct $Z^{0}$ and photon terms and from $\gamma-Z^{0}$ interference. Radiative corrections can be divided into the following components:

- emission of real photons from the initial and/or final state fermions,

- corrections to the $Z^{0}$ and $\gamma$ propagators, consisting of loop diagrams involving any particles which couple to these bosons,

- vertex corrections, involving virtual photons as well as any other particles which couple to the initial or final fermions,

- box diagrams, involving the exchange of two bosons $\left(\gamma, Z^{0}\right)$.

At the present level of precision, the box diagrams can be neglected. To a very good approximation, the effect of the vertex and propagator corrections can be absorbed into a redefinition of the Born-level parameters that retains the structure of the Born-level formulae (Improved or Effective Born Approximation; see e.g. [4]). 
Pure QED initial state corrections can be described by a radiator function $H_{i}\left(s, s^{\prime}\right)$, giving the probability density of emitting a photon with a given fraction of the beam energy from the initial state, such that the observed cross-section for $e^{+} e^{-} \rightarrow f \bar{f}(n \gamma)$ can be written:

$$
\sigma_{o b s}^{f \bar{f}}(s)=\int_{s_{0}^{\prime}}^{s} H_{i}\left(s, s^{\prime}\right) \sigma_{I B}^{f \bar{f}}\left(s^{\prime}\right) d s^{\prime}
$$

where $s_{0}^{\prime}=4 m_{f}^{2}$, the invariant mass $M_{f \bar{f}}$ of the fermion pair produced is given by the effective annihilation energy $\sqrt{s^{\prime}}$, and $\sigma_{I B}^{f \bar{f}}\left(s^{\prime}\right)$ is the Improved Born cross-section. Thus the observed cross-section involves a convolution of $\sigma_{I B}^{f \bar{f}}\left(s^{\prime}\right)$ with the radiator function $H_{i}\left(s, s^{\prime}\right)$. A similar formula can be written for the case where selection criteria are applied to the final state fermions or to the photons produced.

In addition, there is a small contribution to the cross-section from the interference between initial and final state radiation [5]. This interference is very small in the energy region covered by this analysis, $\sqrt{s^{\prime}}<87 \mathrm{GeV}$ and $E_{\gamma}>1 \mathrm{GeV}$, and can be neglected at the present level of precision.

For the case where a pure ISR sample is selected, the number of events $N_{12}$ in a given interval $\left(s_{1}^{\prime}, s_{2}^{\prime}\right)$ can be written as

$$
N_{12}=\mathcal{L} \int_{s_{1}^{\prime}}^{s_{2}^{\prime}} H_{i}\left(s, s^{\prime}\right) \sigma_{I B}^{f \bar{f}}\left(s^{\prime}\right) d s^{\prime}
$$

where $\mathcal{L}$ is the integrated luminosity. Thus, from the known QED radiator function $H_{i}\left(s, s^{\prime}\right)$ (see [6] and references therein) and the measured integrated luminosity $\mathcal{L}$, the Improved Born cross-section $\sigma_{I B}^{f \bar{f}}\left(s^{\prime}\right)$ for energies $\sqrt{s^{\prime}}$ below the nominal LEP energy $\sqrt{s}$ can be determined by measuring the $\sqrt{s^{\prime}}$ spectrum from the invariant mass distribution of the final state fermion pairs.

\subsection{New physics near the zeroes of the $e^{+} e^{-} \rightarrow \mu^{+} \mu^{-}$cross- section}

At tree level, the $e^{+} e^{-} \rightarrow \mu^{+} \mu^{-}$cross-section can be written, apart from radiative corrections and possible new physics, as

$$
\sigma=\sigma_{L L}+\sigma_{R R}+\sigma_{R L}+\sigma_{L R}
$$

where $i(j)$ in $\sigma_{i j}$ stands for the helicity of the incoming $e^{-}$(outgoing $\mu^{-}$) [7]. Within the Standard Model, $\sigma_{L L}$ and $\sigma_{R R}$ have nearby zeroes such that $\sigma_{L L}+\sigma_{R R}$ almost vanishes near $\sqrt{s}=80 \mathrm{GeV}$. This is not the case for $\sigma_{R L}+\sigma_{L R}$, which vanishes at $\sqrt{s}>m_{Z}$. A study of the cross-section sum $\sigma_{L L}+\sigma_{R R}$ around $80 \mathrm{GeV}$ allows a search for new physics in an environment of minimal background.

As shown in [7], due to their angular dependence of the form $(1 \pm \cos \theta)^{2}$, where $\theta$ is the production polar angle of the $\mu^{-}$relative to the $e^{-}$beam direction, the two helicity components of the cross-section, $\sigma_{L L}+\sigma_{R R}$ and $\sigma_{R L}+\sigma_{L R}$, can be extracted from the data by a polynomial projection of the differential cross-section as a function of $\theta$ :

$$
\begin{gathered}
\tilde{\sigma}_{+}^{\mu}=\int_{-C_{M}}^{C_{M}} \frac{d \sigma}{d \cos \theta} \cdot F_{+} \cdot d \cos \theta \\
\tilde{\sigma}_{-}^{\mu}=\int_{-C_{M}}^{C_{M}} \frac{d \sigma}{d \cos \theta} \cdot F_{-} \cdot d \cos \theta,
\end{gathered}
$$


where the filters $F_{ \pm}$are polynomials of the form $A(1 \pm B \cos \theta)$, and $C_{M}=\cos \theta_{\max }$ represents the acceptance limits in the analysis.

At tree level in the framework of the Standard Model,

$$
\begin{aligned}
& \tilde{\sigma}_{+}^{\mu}=\sigma_{L L}+\sigma_{R R} \\
& \tilde{\sigma}_{-}^{\mu}=\sigma_{L R}+\sigma_{R L}
\end{aligned}
$$

and the ratio

$$
\frac{\tilde{\sigma}_{+}^{\mu}}{\tilde{\sigma}_{-}^{\mu}}=\frac{\sigma_{L L}+\sigma_{R R}}{\sigma_{R L}+\sigma_{L R}}
$$

has a minimum just below $80 \mathrm{GeV}$. Therefore, this ratio is very sensitive to new physics near this energy.

\section{The DELPHI detector and data samples}

The DELPHI detector and its performance are described in detail in [8]. In this analysis, the tracking was performed by the Vertex Detector (VD), the Inner Detector (ID), the Outer Detector (OD), the Time Projection Chamber (TPC), and the Forward Chambers (FCA,FCB). In the rejection of events with final state radiation (FSR), the barrel and forward electromagnetic calorimeters were used (HPC and FEMC). The identification of muons was based on the muon chambers (MUB and MUF), the hadron calorimeter (HCAL), and the electromagnetic calorimeters. In what follows, the barrel region was defined as $43^{\circ} \leq \theta \leq 137^{\circ}$, and the endcaps as $\theta<43^{\circ}$ and $\theta>137^{\circ}$, where $\theta$ is the polar angle with respect to the electron beam axis.

The data used for the calculation of the forward-backward asymmetries and the helicity cross-section ratios were collected during the years 1991 to 1994, and correspond to an integrated luminosity of around $115 \mathrm{pb}^{-1}$. For the calculation of the total cross-sections, only the data taken during the years 1992-1994 were used, corresponding to an integrated luminosity of around $100 \mathrm{pb}^{-1}$. About $80 \%$ of the events were collected at the $Z^{0}$ peak energy, while the others were produced during a scan at different energies below and above the $Z^{0}$ peak.

For simulation studies of the signal, dimuon events were generated using the DYMU3 program [9]. The events were generated with up to two initial state photons and at most one final state photon. For the cross-section studies, where a normalisation to the simulated sample is needed, a total of about 613,000 events were generated, spread over the beam energies at which data were taken in the period 1992 to 1994 . For the evaluation of the efficiency and purity of the ISR sample (see Section 4.3), about 240,000 radiative muon events with $\mu^{+} \mu^{-}$invariant mass $M_{\mu \mu}<88 \mathrm{GeV} / \mathrm{c}^{2}$ were generated at the same beam energies.

For the study of the background from the channel $e^{+} e^{-} \rightarrow \tau^{+} \tau^{-}$, about 270,000 events were generated with the KORALZ program [10]. For the study of the background coming from $\gamma \gamma$ processes, about 236,000 $e^{+} e^{-} \rightarrow e^{+} e^{-} \mu^{+} \mu^{-}$and $10,000 e^{+} e^{-} \rightarrow e^{+} e^{-} \tau^{+} \tau^{-}$ events were generated using the FERMISV program [11].

All the generated events were passed through the full DELPHI simulation [8] and the same data reconstruction program as the real data. 


\section{Selection of dimuon events with ISR}

The selection of dimuon events with ISR was performed in two steps. First a sample of dimuon events with or without photon production was selected following the procedure detailed in Section 4.1. From this sample, events with ISR were extracted using the procedure described in Section 4.2 .

For the calculation of the cross-sections, the same selection procedure was applied to the 613,000 simulated dimuon events (see Section 3).

\subsection{Selection of $e^{+} e^{-} \rightarrow \mu^{+} \mu^{-}(n \gamma)$ events}

To select a sample of dimuon events allowing for possible photon emission, the procedure explained in [12] was followed, with looser cuts to admit a larger fraction of radiative events and consequently with a more elaborate rejection of $\tau^{+} \tau^{-}$events.

The events had to contain two charged particles of momentum greater than $10 \mathrm{GeV} / c$, both of which were identified as muons either by the muon chambers (MUB and MUF), or by the hadron calorimeter (HCAL), or by the electromagnetic calorimeters (HPC and FEMC). Both particles had to come from the interaction region, which was defined as $|z|$ less than $4.5 \mathrm{~cm}$ and $R$ less than $1.5 \mathrm{~cm}$, where $z$ is along the $e^{-}$beam direction and $R$

is the radius normal to it. The variable $P_{\text {rad }}=\sqrt{p_{1}^{2}+p_{2}^{2}} / E_{\text {beam }}$, where $E_{\text {beam }}$ is the beam energy and $p_{1}$ and $p_{2}$ are the momenta of the two muons, had to exceed 0.3 . Events with more than 5 charged particle tracks were rejected.

Because these cuts were looser than those used in the standard dimuon selection, the background from $\tau^{+} \tau^{-}$events was higher. To reduce this background, the following three criteria were introduced. Firstly, if the acollinearity angle between the two muons was larger than $1^{\circ}$, the event was rejected if the energy deposited in the HCAL was larger than a cutoff value dependent on the polar angle (see [12]). Secondly, if the event had more than 2 charged particle tracks, either the acollinearity angle between the two muons had to be less than $1^{\circ}$ or both muons had to have at least one associated hit in the muon chambers. Thirdly, in the procedure to separate ISR from Final State Radiation (FSR) events, a variable $\Delta E_{\gamma}$ was introduced, which was defined as

$$
\Delta E_{\gamma}=E_{\gamma}^{\prime}-E_{\gamma}^{\prime \prime}
$$

where

$$
E_{\gamma}^{\prime}=\sqrt{s}-E_{\mu^{+}}-E_{\mu^{-}}
$$

and

$$
E_{\gamma}^{\prime \prime}=\frac{\left|\sin \left(\theta_{\mu^{+}}+\theta_{\mu^{-}}\right)\right|}{\left|\sin \left(\theta_{\mu^{+}}+\theta_{\mu^{-}}\right)\right|+\sin \theta_{\mu^{+}}+\sin \theta_{\mu^{-}}} \sqrt{s} .
$$

In these formulae, $\theta_{\mu^{+}}$and $\theta_{\mu^{-}}$are the polar angles, and $E_{\mu^{+}}$and $E_{\mu^{-}}$the energies of the muons. The variable $E_{\gamma}^{\prime}$ represents the energy taken by the ISR photons if no FSR photons are produced. The variable $E_{\gamma}^{\prime \prime}$ is an approximation to the ISR photon energy if the muon masses are neglected and if one assumes that a single ISR photon is emitted along the beam direction and that no FSR photons are produced. The variable $\Delta E_{\gamma}$ was also effective in rejecting tau events, as can be seen in Figure 1a, which shows the distribution of this variable for simulated dimuon and $\tau^{+} \tau^{-}$events, after application of all of the above cuts except the tau rejection criteria. Events with $\Delta E_{\gamma}>25 \mathrm{GeV}$ were rejected as tau candidates.

Because the selection efficiencies could not be estimated reliably at low polar angles, the cross-sections were determined with samples of events with the $\mu^{-}$polar angle in the 
region $20^{\circ} \leq \theta_{\mu^{-}} \leq 160^{\circ}$. For the measurement of the forward-backward asymmetries however, the likelihood fit method is not affected by the selection efficiencies if these are forward-backward symmetric. Therefore, for these measurements the $\mu^{-}$polar angle region was extended to $11^{\circ} \leq \theta_{\mu^{-}} \leq 169^{\circ}$. For the extraction of the helicity cross-sections, efficiency corrections had to be made, and the polar angle acceptance was dependent on the effective energy $\sqrt{s^{\prime}}$ (see Section 6.3).

After this selection of dimuon events, data runs were rejected if the parts of the DELPHI detector used in this analysis were not fully operational. The total number of dimuons available for the asymmetry analysis (data taking period 1991-1994) amounted to 128,876 . These were spread over 7 energy points, but about $92 \%$ were produced at the $Z^{0}$ peak. The total number of dimuons selected for the cross-section analysis (data taking period 1992-1994) was 107,619 spread over 3 energy points, about $91 \%$ of them being produced at the $Z^{0}$ peak.

The tau background was estimated with the simulated $\tau^{+} \tau^{-}$events to be $0.26 \%$. The background from two-photon events was also estimated with simulated events, and found to be less than $0.1 \%$ for the channel $e^{+} e^{-} \rightarrow e^{+} e^{-} \mu^{+} \mu^{-}$. For the channel $e^{+} e^{-} \rightarrow$ $e^{+} e^{-} \tau^{+} \tau^{-}$, no simulated events were found to satisfy the dimuon selection criteria.

The cosmic ray background was estimated from the data, by relaxing the definition of the interaction region [12] and counting the number of additional events accepted in the data sample. It was found to amount to $0.57 \%$.

From the 613,000 simulated $\mu^{+} \mu^{-}$events, 508,058 remained after the dimuon selection in the polar angle region $20^{\circ} \leq \theta_{\mu^{-}} \leq 160^{\circ}$. These events were used for the normalisation in the cross-section calculation (see Section 5 ).

\subsection{Selection of $e^{+} e^{-} \rightarrow \mu^{+} \mu^{-} \gamma_{\mathrm{ISR}}$ events}

To extract the events with ISR from the dimuon sample described in Section 4.1, the following variables were used.

- $p_{T}^{\text {miss }}=\left|\sum_{i=1}^{N_{c h}} p_{T}^{\vec{i}}\right|$, the missing transverse momentum of the event with respect to the beam axis.

- $\alpha_{\text {acol }}$, the acollinearity angle between the two muons.

- $\alpha_{\text {acop }}$, the acoplanarity angle defined as the angle between the two planes respectively formed by each of the muons and the electron beam axis.

- $E_{e m}^{n e u}$, the total neutral electromagnetic energy of the event, defined as

$$
E_{e m}^{n e u}=E_{e m}^{t o t}-E_{e m}^{\mu^{+}}-E_{e m}^{\mu^{-}}
$$

where $E_{e m}^{\text {tot }}$ is the total energy deposited in the electromagnetic calorimeters and $E_{e m}^{\mu^{+}}$ $\left(E_{e m}^{\mu^{-}}\right)$is the energy deposited by the $\mu^{+}\left(\mu^{-}\right)$.

- $\Delta E_{\gamma}$, defined in equations (9) to (11). Figure 1 b shows the distribution of $\Delta E_{\gamma}$ for simulated ISR and FSR events after applying all the selections mentioned in Section 4.1 except the $\Delta E_{\gamma}$ cut. The events were labelled as ISR or FSR on the basis of the generator information. Figure $1 \mathrm{~b}$ shows that the ISR events are concentrated in the region $\left|\Delta E_{\gamma}\right|<15 \mathrm{GeV}$, while the FSR events are spread over the whole $\Delta E_{\gamma}$ domain.

- $\alpha_{\mu \gamma}$, the isolation angle. For a given reconstructed photon, this was taken to be the smallest of the following angles: the two angles between the muon tracks and the flightline of the photon, and the two angles between the direction opposite to the muons and the flightline of the photon. The photons were assumed to be produced 
at the vertex. They were reconstructed following the method described in [8]. Only photons with a reconstructed energy larger than $1 \mathrm{GeV}$ were considered.

To ensure a high purity of the selected sample for all effective annihilation energies, the selection criteria were taken to be different in each $\sqrt{s^{\prime}}$ interval. The selection criteria are summarised in Table 1.

Two sets of selections were used, called tight or loose, depending on whether or not a photon was detected in the electromagnetic calorimeters close to one of the muons. The tight selections were applied in the barrel region when a photon was found with isolation angle $\alpha_{\mu \nu}<36^{\circ}$, and in the endcaps when either a) a photon was found with isolation angle $\alpha_{\mu \gamma}<20^{\circ}$, or b) $\alpha_{\mu \gamma}<\theta_{\gamma}$, where $\theta_{\gamma}$ is the polar angle of the photon with respect to the electron beam axis. In all other cases, the loose selections were applied.

For the effective annihilation energy $\sqrt{s^{\prime}}$, or equivalently the $\mu^{+} \mu^{-}$invariant mass $M_{\mu \mu}$, the following expression was used:

$$
\sqrt{s^{\prime}}=M_{\mu \mu}=\sqrt{s-2 E_{\gamma}^{\prime \prime} \sqrt{s}}
$$

The justification for this procedure is explained in [2]. Figure 2 shows the relation between the true effective annihilation energy and approximation (13) for simulated ISR events. The mean difference between the true and reconstructed values was found to be around $1 \%$ at $87 \mathrm{GeV}$ and about $7 \%$ at energies below $60 \mathrm{GeV}$. The analysis was restricted to the $\sqrt{s^{\prime}}$ region between 20 and $87 \mathrm{GeV}$.

In the data sample, 325 ISR events were selected for the cross-section calculation, and 399 for the differential cross-section and asymmetry calculation.

From the 508,058 simulated dimuons selected as described in Section 4.1, 1868 ISR events were selected for the cross-section calculations.

\subsection{Efficiency of the selection procedure and purity of the ISR sample}

The efficiency of the selection procedure and the contamination by FSR events were studied with the sample of simulated radiative muon events generated by DYMU3 [9] with true $\sqrt{s^{\prime}}$ lower than $88 \mathrm{GeV}$. The events were flagged as ISR or FSR on the basis of the generator information. If both initial and final state photons were produced, the events were labelled ISR if the generated final state photon energy was below $1 \mathrm{GeV}$ and FSR otherwise. The effective annihilation energy $\sqrt{s^{\prime}}$ was calculated using formula (13). The resulting efficiency and purity are displayed as a function of $\sqrt{s^{\prime}}$ in Figure 3 . The purity of the sample is near $90 \%$ over the whole energy interval. The selection efficiency is about $80 \%$ at low energies and decreases to around $50 \%$ at $87 \mathrm{GeV}$.

The cosmic ray background was checked using the sample of ISR events selected for the cross-section calculation. No additional events were found when the cuts on the interaction region definition were relaxed to allow impact parameters of charged particle tracks to the production vertex of up to $3 \mathrm{~cm}$ in the plane transverse to the beam and up to $9 \mathrm{~cm}$ parallel to the beam, which should have quadrupled this background.

The background from $e^{+} e^{-} \rightarrow \tau^{+} \tau^{-}$events was estimated from the sample of 270,000 simulated $\tau^{+} \tau^{-}$events (see Section 3 ). No events were found to satisfy the ISR selection criteria.

The background from two-photon processes was estimated from simulated events. It was found to be $1.6 \%$ for the channel $e^{+} e^{-} \rightarrow e^{+} e^{-} \mu^{+} \mu^{-}$, while no events in the channel $e^{+} e^{-} \rightarrow e^{+} e^{-} \tau^{+} \tau^{-}$satisfied the ISR criteria. 


\section{Estimation of the total Improved Born cross- sections}

The data taken in the years 1992-1994 were used to estimate the Improved Born cross-sections $\sigma_{I B}^{o b s}\left(\sqrt{s^{\prime}}\right)$. The polar angle of the $\mu^{-}$was required to be in the range $20^{\circ} \leq \theta_{\mu^{-}} \leq 160^{\circ}$. A total of 325 events was selected. The ratio of the averages of the observed to the Standard Model Improved Born cross-sections inside a given $\sqrt{s^{\prime}}$ interval is given by

$$
\frac{\left\langle\sigma_{I B}^{o b s}\left(\sqrt{s^{\prime}}\right)\right\rangle}{\left\langle\sigma_{I B}^{S M}\left(\sqrt{s^{\prime}}\right)\right\rangle}=\frac{N_{o b s}\left(\sqrt{s^{\prime}}\right) \cdot N_{s i m}^{n o r m}}{N_{\text {sim }}\left(\sqrt{s^{\prime}}\right) \cdot N_{o b s}^{n o r m}}
$$

where $\sqrt{s^{\prime}}$ is the mean effective annihilation energy in the interval. The quantities $N_{\text {obs }}\left(\sqrt{s^{\prime}}\right)$ and $N_{\text {sim }}\left(\sqrt{s^{\prime}}\right)$ represent the numbers of ISR events in a given $\sqrt{s^{\prime}}$ interval in the data and in the simulated sample respectively, selected as explained in Section 4.2. The quantities $N_{o b s}^{\text {norm }}$ and $N_{\text {sim }}^{\text {norm }}$ represent the number of dimuon events selected as explained in Section 4.1 in the real and simulated data samples. In each $\sqrt{s^{\prime}}$ interval, the normalisation of the ISR sample to the full dimuon sample was calculated separately for the on-peak and off-peak data, after which the results were averaged. The number $N_{\text {obs }}\left(\sqrt{s^{\prime}}\right)$ was corrected for the two-photon background, and the number $N_{o b s}^{n o r m}$ was corrected for the background arising from cosmic ray and tau events. The other backgrounds were too small to justify a correction. It was verified that the selection efficiency for ISR events was the same for the observed data and the simulation.

The theoretical Improved Born cross-section, $\sigma_{I B}^{S M}\left(\sqrt{s^{\prime}}\right)$, was obtained from the DYMU3 program. The parameters used in this calculation were $M_{Z}=91.25 \mathrm{GeV} / \mathrm{c}^{2}$, $\Gamma_{Z}=2.562 \mathrm{GeV} / \mathrm{c}^{2}$, and $\sin ^{2} \theta_{W}=0.2296$, which were the default values used by DELPHI for the generation of $\mu^{+} \mu^{-}$events. It was shown previously [2] that the calculations made by this program and those made by the ZFITTER program [6] agreed to within $1 \%$.

Table 2 shows the number of ISR events selected in the data and in the simulated samples as a function of $\sqrt{s^{\prime}}$, up to an energy of $87 \mathrm{GeV} / \mathrm{c}^{2}$. The same table shows the calculated SM Improved Born cross-section in the different $\sqrt{s^{\prime}}$ intervals as well as the experimental cross-section obtained from formula (14). Only statistical errors were taken into account. The main source of systematic errors was the modelling of the muon momenta in the simulation. To reduce these effects to a negligible size, the muon momenta were smeared in the simulation to match the resolution observed in the data.

Figure 4 shows the ratios between the observed and theoretical Improved Born crosssections as a function of the effective annihilation energy, and the resulting experimental cross-sections. It also shows the cross-sections for the reaction $e^{+} e^{-} \rightarrow \mu^{+} \mu^{-}$obtained near the $Z^{0}$ peak [12,14] and those obtained at PEP, PETRA, and TRISTAN [15,1]. The published values were corrected to obtain Improved Born values. The cross-section measured with the VENUS detector at $58 \mathrm{GeV}$ has a value of $29.07 \pm 0.66 \mathrm{pb}$ [1], which has to be compared with the Standard Model prediction of $30.72 \mathrm{pb}$. The resolution of Figure 4 is not sufficient to show this deviation. 


\section{$6 \quad$ Asymmetries and helicity cross-sections}

\subsection{Polar angle distributions}

The data taken in the years 1991-1994 were used to obtain the distributions of the muon polar angle in the $\mu^{+} \mu^{-}$rest frame for different $\sqrt{s^{\prime}}$ intervals. A total of 399 events were selected. The distribution of these events as a function of $\sqrt{s^{\prime}}$ is shown in Table 3 .

For events which are not produced in the $e^{+} e^{-} \mathrm{c.m}$. frame, the angle between the $\mu^{-}$ and the $e^{-}$beam direction in the $\mu^{-} \mu^{+}$rest frame is given by [13]:

$$
\cos \theta^{*}=\frac{\sin \frac{1}{2}\left(\theta_{\mu^{+}}-\theta_{\mu^{-}}\right)}{\sin \frac{1}{2}\left(\theta_{\mu^{+}}+\theta_{\mu^{-}}\right)}
$$

where $\theta_{\mu^{+}}$and $\theta_{\mu^{-}}$are the polar angles of the $\mu^{+}$and the $\mu^{-}$with respect to the $e^{-}$beam axis in the laboratory frame.

The raw distributions of $\cos \theta^{*}$ in the different $\sqrt{s^{\prime}}$ intervals are shown in Figure 5 . They were corrected for selection inefficiencies in the following way. It was assumed that, within the limits of the present statistics, forward-backward asymmetries in detection efficiency could be neglected. Indeed, such asymmetries distort the forward-backward asymmetry by at most 0.001 [14]. Therefore, in each $\sqrt{s^{\prime}}$ interval of the data, the correction factor was obtained from the folded distribution in $\left|\cos \theta^{*}\right|$, which was fitted to a function of the form

$$
\frac{d N_{r}}{d\left|\cos \theta^{*}\right|}=\frac{3}{8} C\left(1+\cos ^{2} \theta^{*}\right)
$$

in the region of $\left|\cos \theta^{*}\right|$ where the fit gave the smallest value of $\chi^{2} / N_{d}$. The $C$ value obtained from this fit was used to calculate correction factors in the $\left|\cos \theta^{*}\right|$ bins outside the fit region, namely $F\left(\left|\cos \theta^{*}\right|\right)=\frac{3}{8} C\left(1+\cos ^{2} \theta^{*}\right) / N_{r}\left(\left|\cos \theta^{*}\right|\right)$, where $N_{r}$ is the raw number of events. In the unfolded $\cos \theta^{*}$ distribution, the raw bin contents for positive and negative $\cos \theta^{*}$ were corrected with the same correction factors $F\left(\left|\cos \theta^{*}\right|\right)$. The resulting corrected $\cos \theta^{*}$ distributions are also shown in Figure 5 for the five $\sqrt{s^{\prime}}$ intervals considered.

In each $\sqrt{s^{\prime}}$ interval, the $\cos \theta^{*}$ distribution was then corrected for the background coming from FSR, which on average amounted to about $10 \%$. In each interval, a distribution of the form

$$
\frac{d N_{F S R}}{d \cos \theta^{*}}=C^{\prime}\left[\frac{3}{8}\left(1+\cos ^{2} \theta^{*}\right)+A_{f b}^{0} \cos \theta^{*}\right]
$$

was subtracted, where $A_{f b}^{0}$ was taken to be the asymmetry at the $Z^{0}$ peak, from which this background dominantly arises. The constants $C^{\prime}$ were obtained from the integral of equation (17), which is equal to the expected number of FSR events in a given $\sqrt{s^{\prime}}$ interval as predicted by the simulated radiative muon sample. The contamination by tau events, cosmics or two-photon events was too small to justify a correction.

\subsection{Forward-backward asymmetries}

In each $\sqrt{s^{\prime}}$ interval, the asymmetry $A_{F B}$ was obtained by performing a maximum likelihood fit of the uncorrected $\cos \theta^{*}$ distribution to an expression of the form

$$
\frac{d N}{d \cos \theta^{*}}=C^{\prime \prime}\left[\left(1+\cos ^{2} \theta^{*}\right)+\frac{8}{3} A_{F B} \cos \theta^{*}\right] .
$$


Formula (18) does not include radiative corrections. Since the asymmetries determined in this analysis are Improved Born asymmetries, only the electro-weak corrections should be considered. Compared to the experimental precision they are small, and modify the asymmetry by at most 0.02 in the energy region between 40 and $88 \mathrm{GeV}$.

The asymmetry was also calculated, after correcting for the selection inefficiency as described in Section 6.1, by counting the number of events with positive and negative $\cos \theta^{*}$, since

$$
A_{F B}=\frac{N_{F}-N_{B}}{N_{F}+N_{B}}
$$

with

$$
N_{F}=\int_{0}^{1} \frac{d N}{d \cos \theta^{*}} d \cos \theta^{*} \quad N_{B}=\int_{-1}^{0} \frac{d N}{d \cos \theta^{*}} d \cos \theta^{*} .
$$

Table 3 shows the asymmetries obtained with the two methods as a function of $\sqrt{s^{\prime}}$ up to $87 \mathrm{GeV}$.

The raw asymmetries obtained with the fit were corrected for the contamination by FSR events in a similar way as the polar angle distributions were corrected (see Section 6.1). These corrected values are also shown in Table 3, and are displayed in Figure 6 together with the SM prediction for the Improved Born asymmetry. Figure 6 also shows the asymmetries measured by DELPHI near the $Z^{0}$ peak (see $[12,14]$ ), after correction to Improved Born values. The SM Improved Born asymmetry was calculated with the DYMU3 program with the parameters mentioned in Section 5. The only source of systematic error on the asymmetry, $\delta\left(A_{F B}\right)_{\text {sys }}^{\text {fit }}$, which was considered was that resulting from the error on the purity. The values of this error are shown in Table 3.

\subsection{Extraction of the helicity cross-sections and test of the Stan- dard Model.}

From the $\cos \theta^{*}$ distributions corrected for selection efficiencies and for the FSR background, the cross-sections $\tilde{\sigma}_{+}^{\mu}$ and $\tilde{\sigma}_{-}^{\mu}$ (see Section 2.2) and their ratio were determined as follows. In each bin of the $\cos \theta^{*}$ distribution the corrected content was multiplied by a weight factor

$$
F_{ \pm}=A\left(1 \pm B \cos \theta^{*}\right)
$$

where

$$
A=\frac{2}{C_{M}\left(3+C_{M}^{2}\right)} \quad B=\frac{3+C_{M}^{2}}{2 C_{M}^{2}}
$$

and $C_{M}=\cos \theta_{\text {max }}^{*}$. These weighted contents were summed for all $\cos \theta^{*}$ bins between -0.8 and +0.8 for $\sqrt{s^{\prime}}$ below $65 \mathrm{GeV}$, and between -0.9 and +0.9 for the other $\sqrt{s^{\prime}}$ values. The $C_{M}$ limits were chosen to match the binning in each $\sqrt{s^{\prime}}$ interval. The values of $\tilde{\sigma}_{+}^{\mu}$ and $\tilde{\sigma}_{-}^{\mu}$ and their statistical errors were derived from the weighted sums. For the systematic error, the error on the purity of the sample was taken into account. The numbers obtained are given as a function of $\sqrt{s^{\prime}}$ in Table 3 and are shown in Figure 7 , together with the prediction of the Standard Model. The theoretical predictions include all electroweak radiative corrections, apart from the box diagrams which are very small (see [7]). There is no indication of any deviation from the Standard Model. 


\section{Summary}

Using the data collected by DELPHI until 1994, a sample of around 400 dimuon events with initial state radiation was selected. The differential and total cross-sections and forward-backward asymmetries were determined at energies between 20 and $87 \mathrm{GeV}$. No deviation from the Standard Model was observed.

The polar angle distributions were used to determine the ratio $\frac{\sigma_{L L}+\sigma_{R R}}{\sigma_{R L}+\sigma_{L R}}$, where the two subscripts represent the helicities of the incoming $e^{-}$and outgoing $\mu^{-}$respectively. The aim was to test the Standard Model near the minimum of this ratio, around $80 \mathrm{GeV}$, where the sensitivity to new physics is greatest. No evidence for new physics was seen.

\section{Acknowledgements}

We are greatly indebted to our technical collaborators and to the funding agencies for their support in building and operating the DELPHI detector, and to the members of the CERN-SL Division for the excellent performance of the LEP collider.

We would like to thank J.-M. Frêre for suggesting the analysis in terms of helicity cross-sections, and for many fruitful discussions. 


\section{References}

[1] K. Miyabayashi, Recent electroweak results from TRISTAN, Proc. of the XXXth Rencontres de Moriond, Les Arcs, March 1995, ed. J. Trân Thanh Vân.

[2] P. Abreu at al., DELPHI Collab., Z. Phys. C65 (1995) 603.

[3] D. Schaile, Precision tests of the electroweak interaction, Proc. of the XXVIIth Int. Conf. on High Energy Physics, Glasgow, July 1994, ed. P.J. Bussey and I.G. Knowles, IOP publishing (1995) 27.

[4] F. Berends, Z lineshape, in Z Physics at LEP I, CERN 89-08, Volume 1 (1989).

[5] D. Bardin et al., Nucl. Phys. B351 (1991) 1;

P. Abreu et al., DELPHI Collab., Z. Phys. C72 (1996) 31.

[6] D. Bardin et al., ZFITTER, An Analytical Program for Fermion Pair Production in $e^{+} e^{-}$Annihilation, CERN-TH. 6443/92 and hep-ph/9412201.

[7] J.-M. Frêre, V.A. Novikov, M.I. Vysotsky, Phys. Lett. B386 (1996) 437.

[8] P. Aarnio et al., DELPHI Collab., Nucl. Instr. \& Meth. A303 (1991) 233;

P. Abreu et al., DELPHI Collab., Nucl. Instr. \& Meth. A378 (1996) 57.

[9] J.E. Campagne and R. Zitoun, Z. Phys. C43 (1989) 469;

and Proc. of the Brighton Workshop on Radiative Corrections, Sussex, July 1989.

[10] S. Jadach and Z. Was, Comp. Phys. Com. 36 (1985) 191;

S. Jadach, B.F. Ward and Z. Was, Comp. Phys. Com. 66 (1991) 276.

[11] J. Hilgart, R. Kleis, Comp. Phys. Com. 75 (1993) 191.

[12] P. Abreu et al., DELPHI Collab., Nucl. Phys. B417 (1994) 3.

[13] Z. Was and S. Jadach, Phys. Rev. D41 (1990) 1425.

[14] P. Abreu et al., DELPHI Collab., Nucl. Phys. B418 (1994) 403.

[15] HRS Collab., M. Derrick et al., Phys. Rev. D31 (1985) 2352;

MAC Collab., W. W. Ash et al., Phys. Rev. Lett. 55 (1985) 1831;

TASSO Collab., W. Braunschweig et al., Z. Phys. C40 (1988) 163;

CELLO Collab., H.-J. Behrend et al., Phys. Lett. B191 (1987) 209;

JADE Collab., W. Bartel et al., Z. Phys. C26 (1985) 507;

MARK J Collab., B. Adeva et al., Phys. Rev. D38 (1988) 2665;

AMY Collab., A. Bacala et al., Phys. Lett. B218 (1989) 112;

AMY Collab., C. Velissaris et al., Phys. Lett. B331 (1994) 227;

TOPAZ Collab., B. Howell et al., Phys. Lett. B291 (1992) 206. 


\begin{tabular}{|c||ccccc|}
\hline$\sqrt{s^{\prime}}[\mathrm{GeV}]$ & $<35$ & $35-65$ & $65-80$ & $80-84$ & $>84$ \\
\hline \hline \multicolumn{7}{|c|}{ loose selections } \\
\hline$p_{T}^{\text {miss }}[\mathrm{GeV} / c]$ & - & $<5$ & $<4$ & $<4$ & $<3$ \\
$\alpha_{\text {acol }}\left[{ }^{\circ}\right]$ & $>1.5$ & $>1.5$ & $>1.5$ & $>1$ & $>1$ \\
$\alpha_{\text {acop }}\left[{ }^{\circ}\right]$ & $<7.5$ & $<7.5$ & $<5$ & $<4$ & $<4$ \\
$E_{\text {em }}^{\text {neu }}[\mathrm{GeV}]$ & - & $<2$ & $<2$ & $<2$ & $<2$ \\
$\left|\Delta E_{\gamma}\right|[\mathrm{GeV}]$ & $<25$ & $<13.2$ & $<6$ & $<6$ & $<5$ \\
\hline \hline \multicolumn{7}{|c|}{ tight selections } \\
\hline$p_{T}^{\text {miss }}[\mathrm{GeV} / c]$ & - & $<2.5$ & $<1$ & $<1$ & $<1$ \\
$\alpha_{\text {acol }}\left[{ }^{\circ}\right]$ & $>3$ & $>3$ & $>3$ & $>2$ & $>2$ \\
$\alpha_{\text {acop }}\left[{ }^{\circ}\right]$ & $<5$ & $<5$ & $<4$ & $<3$ & $<3$ \\
$E_{\text {em }}^{\text {neu }}[\mathrm{GeV}]$ & - & $<1$ & $<1$ & $<1$ & $<1$ \\
$\left|\Delta E_{\gamma}\right|[\mathrm{GeV}]$ & $<25$ & $<4.4$ & $<3$ & $<3$ & $<2.5$ \\
\hline
\end{tabular}

Table 1: Requirements for the selection of ISR events. The details are given in the text.

\begin{tabular}{|c||ccccccccc|}
\hline$\sqrt{s^{\prime}}[\mathrm{GeV}]$ & $24-38$ & $38-45$ & $45-52$ & $52-59$ & $59-66$ & $66-73$ & $73-80$ & $80-84$ & $84-87$ \\
\hline$N_{\text {obs }}$ & 6 & 12 & 12 & 10 & 11 & 21 & 38 & 55 & 160 \\
$N_{\text {sim }}$ & 25 & 79 & 70 & 86 & 94 & 105 & 222 & 338 & 849 \\
$\left\langle\sigma_{I B}^{\text {obs }}\right\rangle /\left\langle\sigma_{I B}^{S M}>\right.$ & 1.20 & 0.84 & 1.11 & 0.70 & 0.54 & 1.02 & 0.92 & 0.92 & 1.14 \\
& \pm 0.56 & \pm 0.26 & \pm 0.36 & \pm 0.23 & \pm 0.19 & \pm 0.25 & \pm 0.16 & \pm 0.12 & \pm 0.07 \\
\hline$<\sqrt{s^{\prime}}>[\mathrm{GeV}]$ & 33.8 & 42.4 & 47.4 & 55.8 & 62.5 & 70.2 & 77.6 & 82.6 & 85.8 \\
$\sigma_{I B}^{S M}[\mathrm{pb}]$ & 85. & 55. & 44. & 33. & 27. & 25. & 30. & 51. & 106. \\
\hline$\sigma_{I B}^{\text {obs }}[\mathrm{pb}]$ & 102. & 46. & 49. & 23. & 15. & 25. & 28. & 47. & 121. \\
$\delta\left(\sigma_{I B}^{\text {obs }}\right)[\mathrm{pb}]$ & \pm 48. & \pm 14. & \pm 16. & \pm 8. & \pm 5. & \pm 6. & \pm 5. & \pm 6. & \pm 8. \\
\hline
\end{tabular}

Table 2: Numbers of ISR events found in the data and simulated samples ( $N_{o b s}$ and $N_{\text {sim }}$ ) for different $\sqrt{s^{\prime}}$ intervals, and the ratios of the average measured to SM Improved Born cross-sections. Then $\left\langle\sqrt{s^{\prime}}\right\rangle$ is the mean measured effective annihilation energy in the interval, and $\sigma_{I B}^{S M}$ is the mean Improved Born cross-section within each energy interval expected in the Standard Model, obtained from the DYMU3 program. Finally $\sigma_{I B}^{o b s}$ with its error $\delta\left(\sigma_{I B}^{o b s}\right)$ is the resulting measured cross-section. 


\begin{tabular}{|c||ccccc|}
\hline$\sqrt{s^{\prime}}[\mathrm{GeV}]$ & $20-50$ & $50-65$ & $65-80$ & $80-84$ & $84-87$ \\
\hline$N_{F}$ & 20 & 10 & 12 & 17 & 55 \\
$N_{B}$ & 19 & 19 & 59 & 53 & 135 \\
\hline$<\sqrt{s^{\prime}}>[\mathrm{GeV}]$ & 43.0 & 59.0 & 74.7 & 82.4 & 85.8 \\
$A_{F B}^{\text {counting }}[\%]$ & $3.4 \pm 15.8$ & $-32.6 \pm 17.6$ & $-69.5 \pm 8.5$ & $-54.0 \pm 10.1$ & $-44.2 \pm 6.5$ \\
$A_{F B}^{f i t}$ unc $[\%]$ & $3.3 \pm 15.8$ & $-45.0 \pm 17.7$ & $-63.0 \pm 7.4$ & $-60.5 \pm 8.9$ & $-41.6 \pm 6.4$ \\
$A_{F B}^{\text {fit }}$ corr $[\%]$ & $3.2 \pm 16.7$ & $-48.9 \pm 19.2$ & $-70.3 \pm 8.2$ & $-66.8 \pm 9.8$ & $-46.2 \pm 7.1$ \\
$\delta\left(A_{F B}\right)_{\text {sys }}^{f i t}[\%]$ & \pm 0.4 & \pm 0.6 & \pm 0.7 & \pm 0.6 & \pm 0.3 \\
\hline \hline$\tilde{\sigma}_{+}^{\mu} / \tilde{\sigma}_{-}^{\mu}$ & $1.20 \pm 0.46$ & $0.24 \pm 0.15$ & $0.05 \pm 0.07$ & $0.10 \pm 0.06$ & $0.23 \pm 0.06$ \\
$\delta_{\text {sys }}$ & 0.05 & 0.01 & 0.01 & 0.01 & 0.01 \\
\hline
\end{tabular}

Table 3:

$N_{F}, N_{B}$ : observed number of ISR events in the forward and backward hemispheres; $A_{F B}^{\text {counting }}$ : the asymmetry with statistical error calculated with the counting method; $A_{F B}^{f i t} u n c:$ the asymmetry with statistical error calculated with a maximum likelihood fit, without correction for FSR contamination;

$A_{F B}^{f i t} \operatorname{corr}$ : the asymmetry with statistical error calculated with a maximum likelihood fit, after correction for FSR contamination, with systematic error $\delta\left(A_{F B}\right)_{s y s}^{f i t}$;

$\tilde{\sigma}_{+}^{\mu} / \tilde{\sigma}_{-}^{\mu}$ : the helicity component ratio defined in Section 2.2, with its statistical error ;

$\delta_{\text {sys }}$ : the systematic error on the helicity component ratio. 

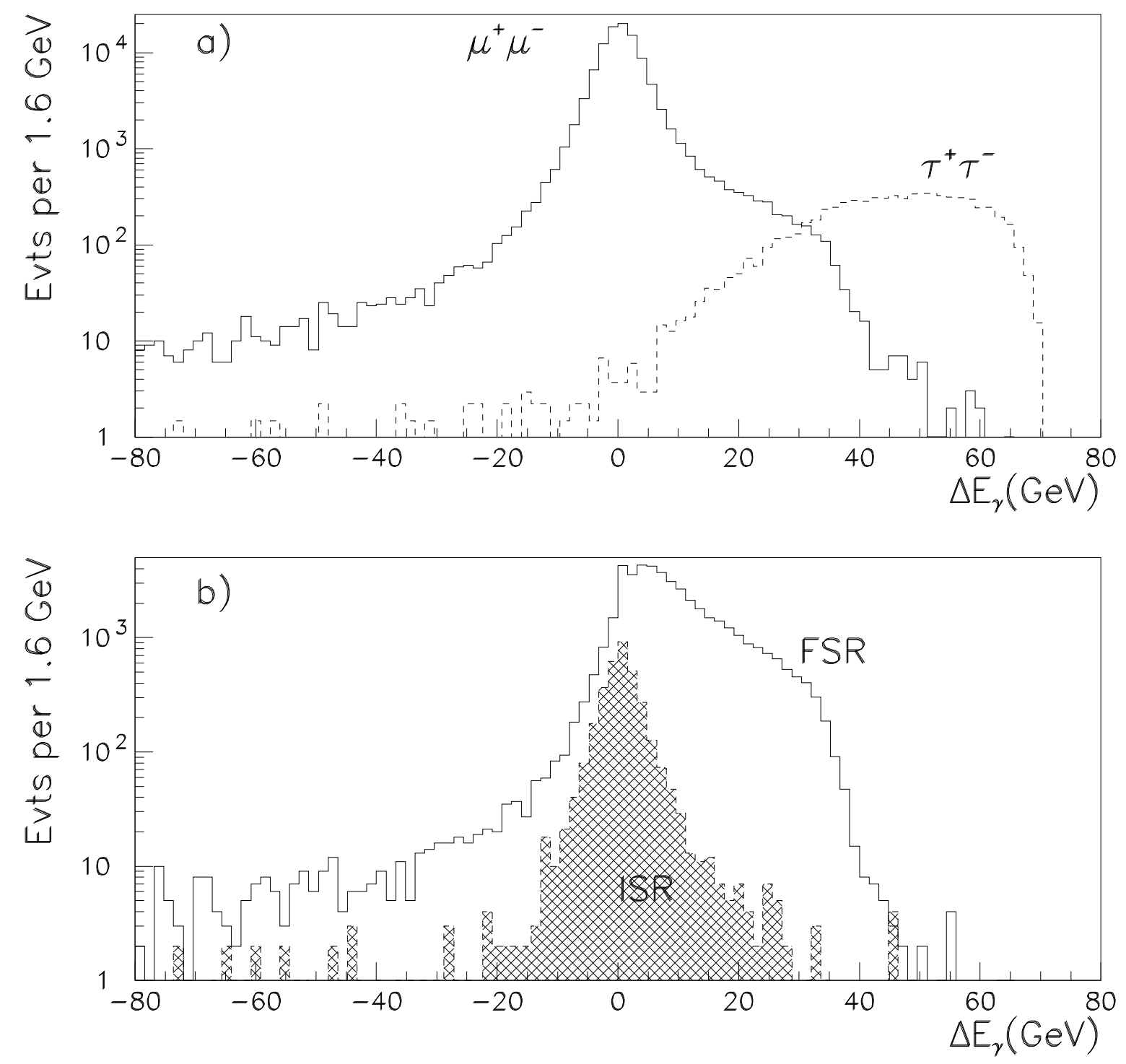

Figure 1: Distribution of $\Delta E_{\gamma}$ (see Section 4.1 for definition) for a) simulated dimuon events (solid line) and $\tau^{+} \tau^{-}$events (dashed line) after all cuts from Section 4.1 except the tau vetoes, and $\mathbf{b}$ ) simulated ISR (cross-hatched area) and FSR events (solid line) with $\sqrt{s^{\prime}}<88 \mathrm{GeV}$ after the selection procedure of Section 4.1 without the $\Delta E_{\gamma}$ cut. 


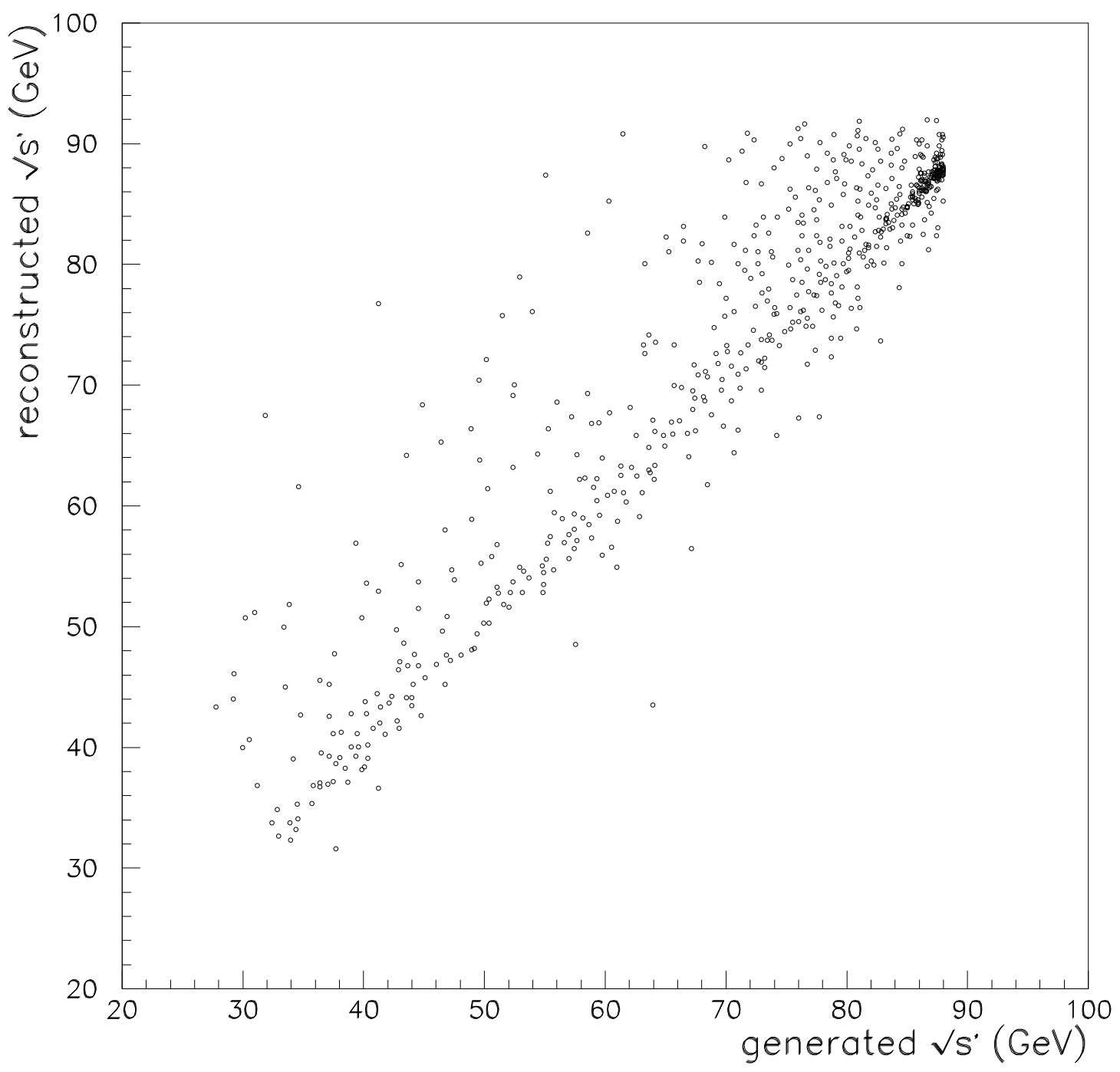

Figure 2: Reconstructed effective annihilation energy $\sqrt{s^{\prime}}$ (formula 13) as a function of the true value for simulated ISR events with true $\sqrt{s^{\prime}}<88 \mathrm{GeV}$. 

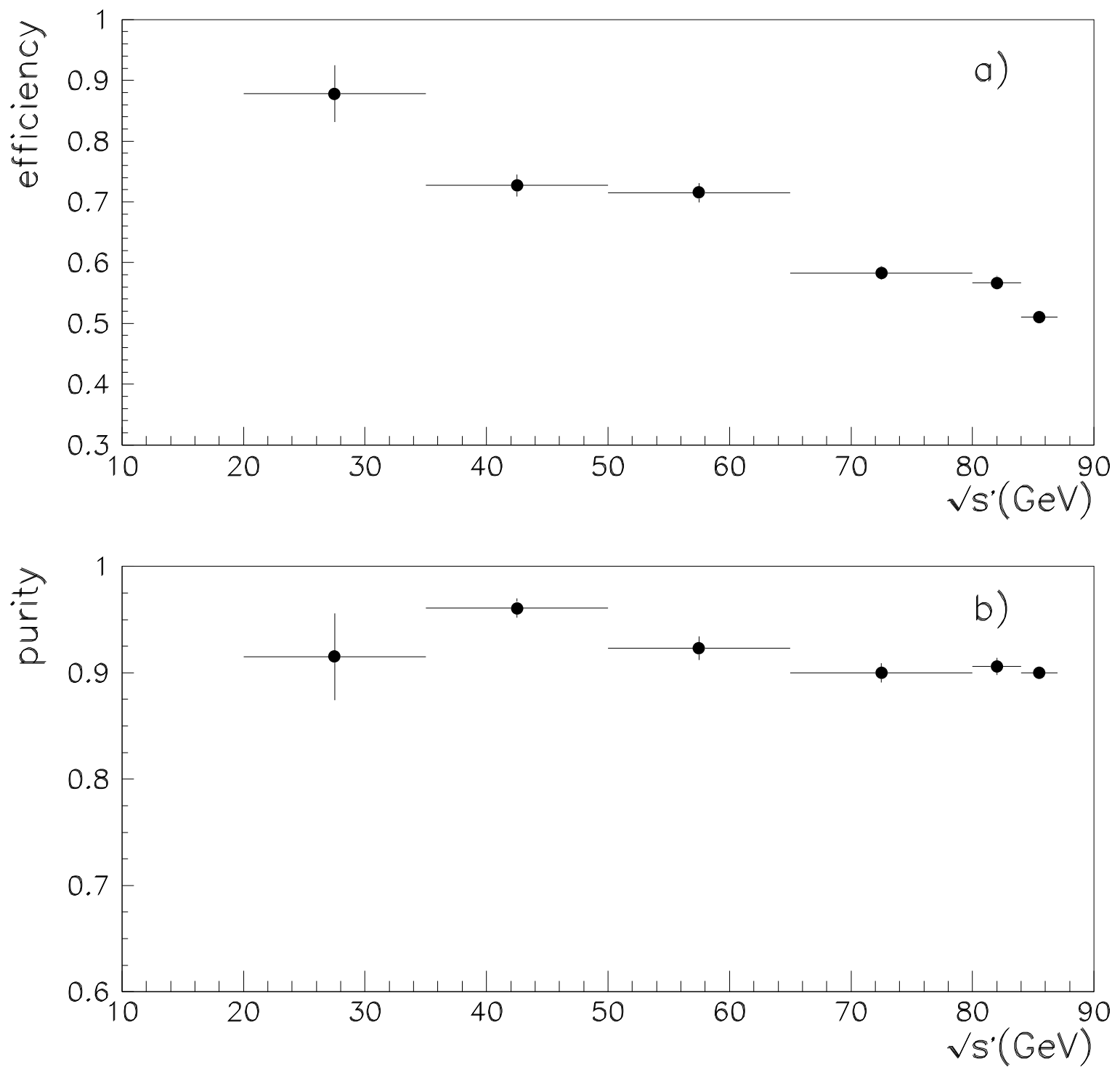

Figure 3: (a) Efficiency for the selection of ISR events and (b) purity of the ISR sample with regard to FSR events, based on simulated radiative muon events with $\sqrt{s^{\prime}}<88 \mathrm{GeV}$. 


\section{DELPHI}
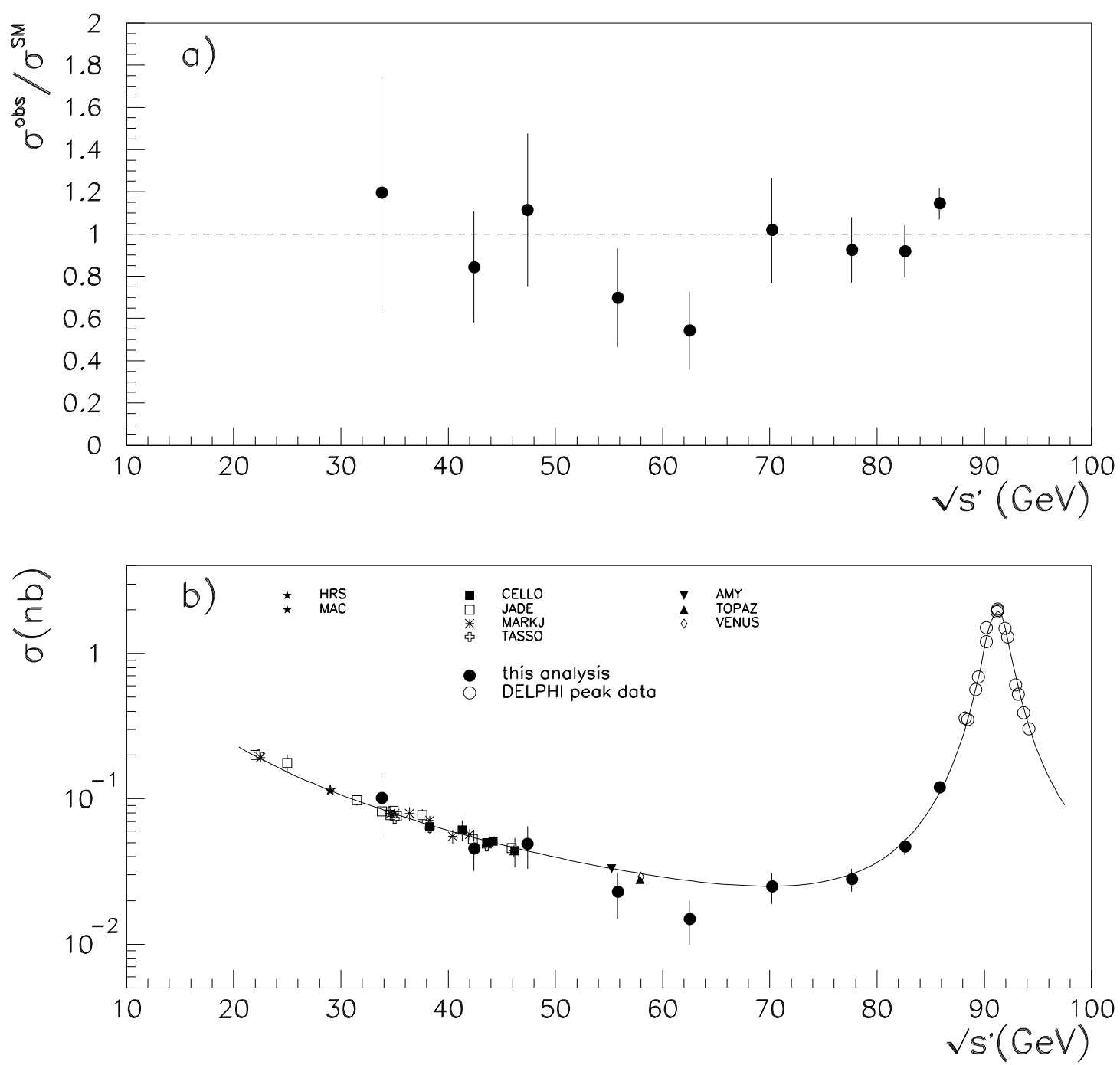

Figure 4: (a) Ratio of measured to SM Improved Born cross-section as a function of the effective annihilation energy. (b) Improved Born cross-sections measured in this analysis (black circles), at the $Z^{0}$ peak (open circles), and at PEP, PETRA and TRISTAN. The solid line shows the SM prediction. 

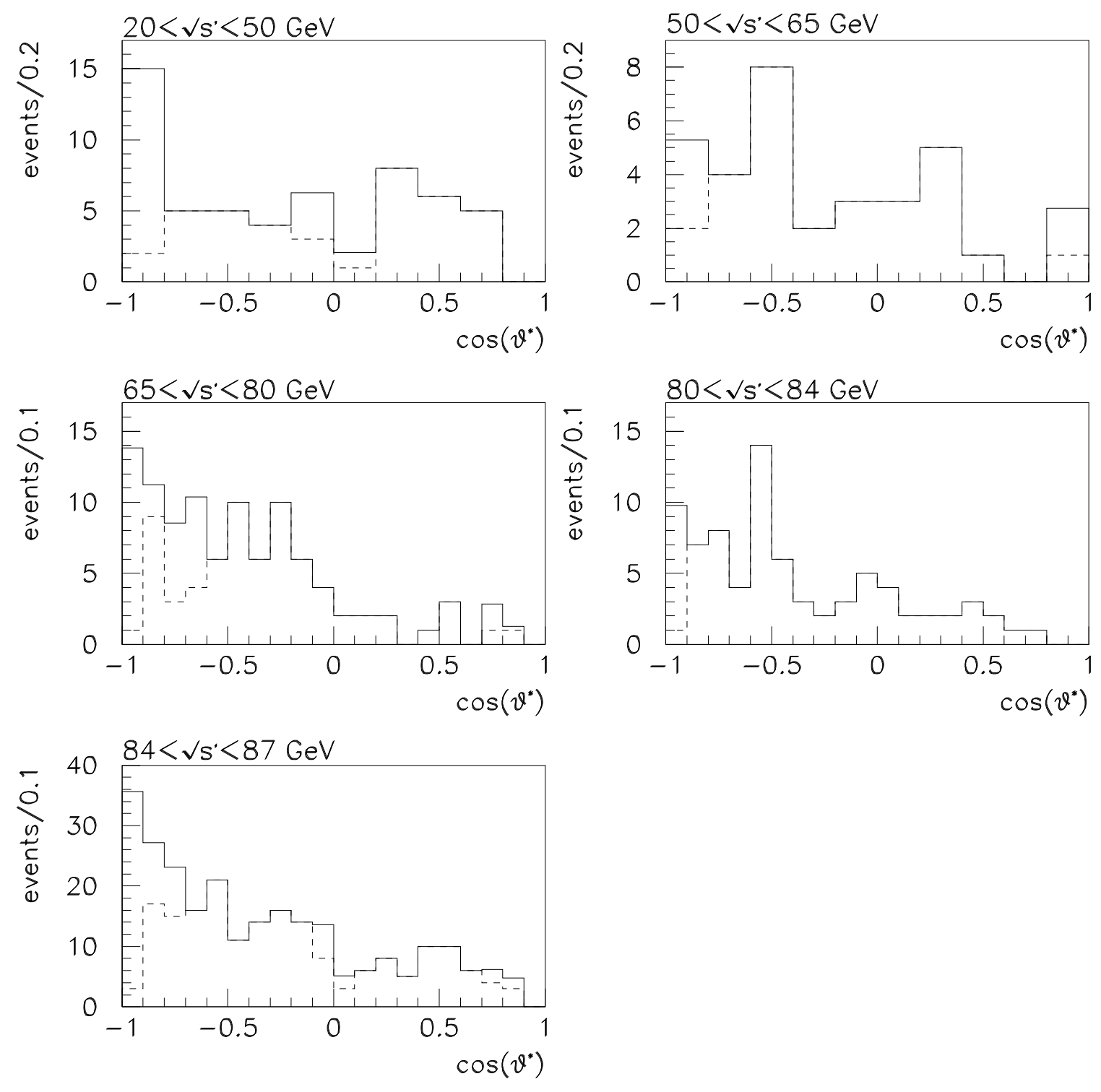

Figure 5: Distribution of $\cos \theta^{*}$ in different intervals of the effective annihilation energy $\sqrt{s^{\prime}}$. The dashed lines show the raw distributions and the solid lines the distributions after correction for the selection efficiency. 


\section{DELPHI}

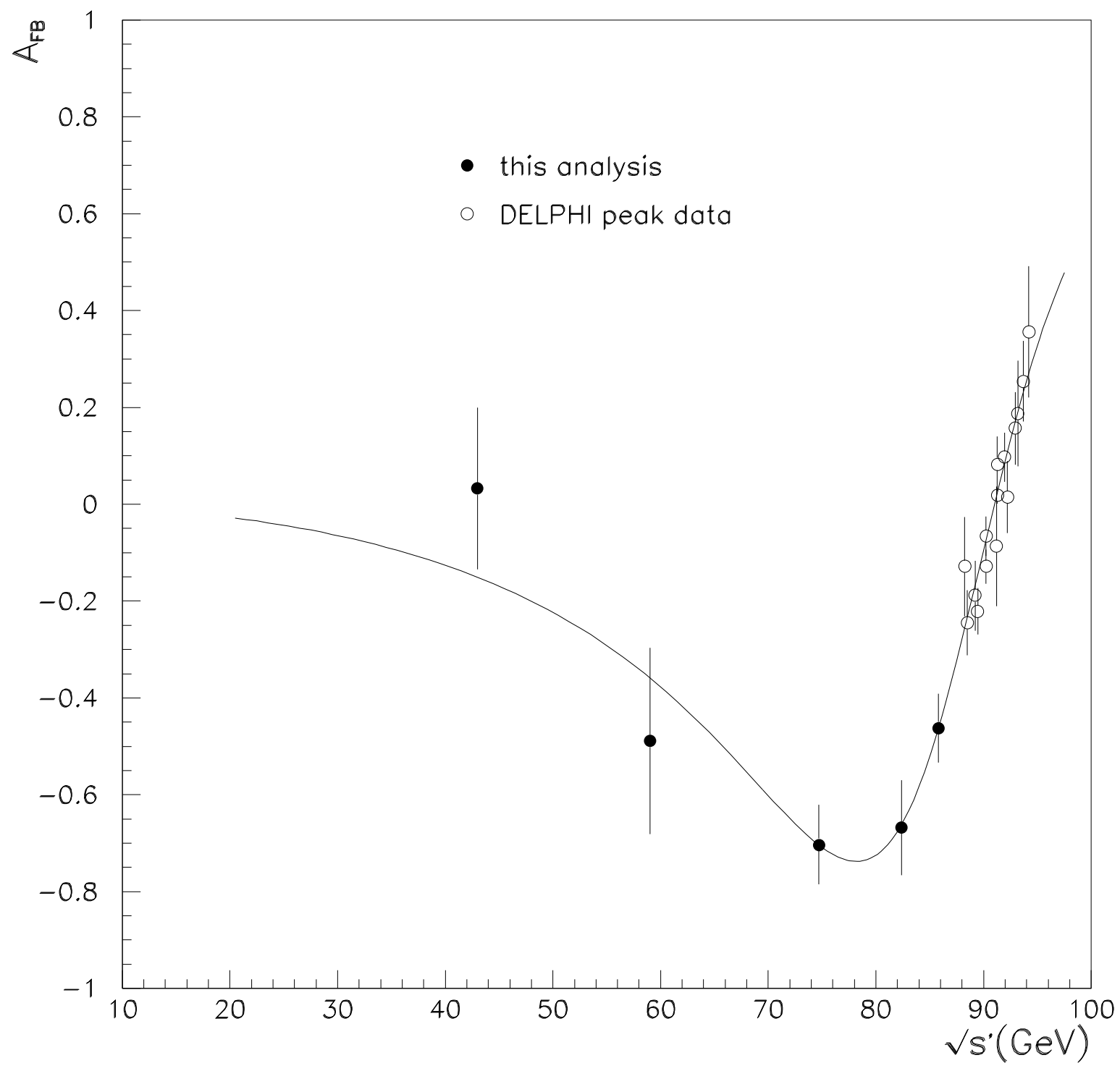

Figure 6: Improved Born asymmetry as a function of the effective annihilation energy. The black points show the measurements made by DELPHI below $87 \mathrm{GeV}$, using the likelihood fit method described in the text. The open circles show the measurements made by DELPHI at the $Z^{0}$ peak. The solid line shows the SM prediction. 


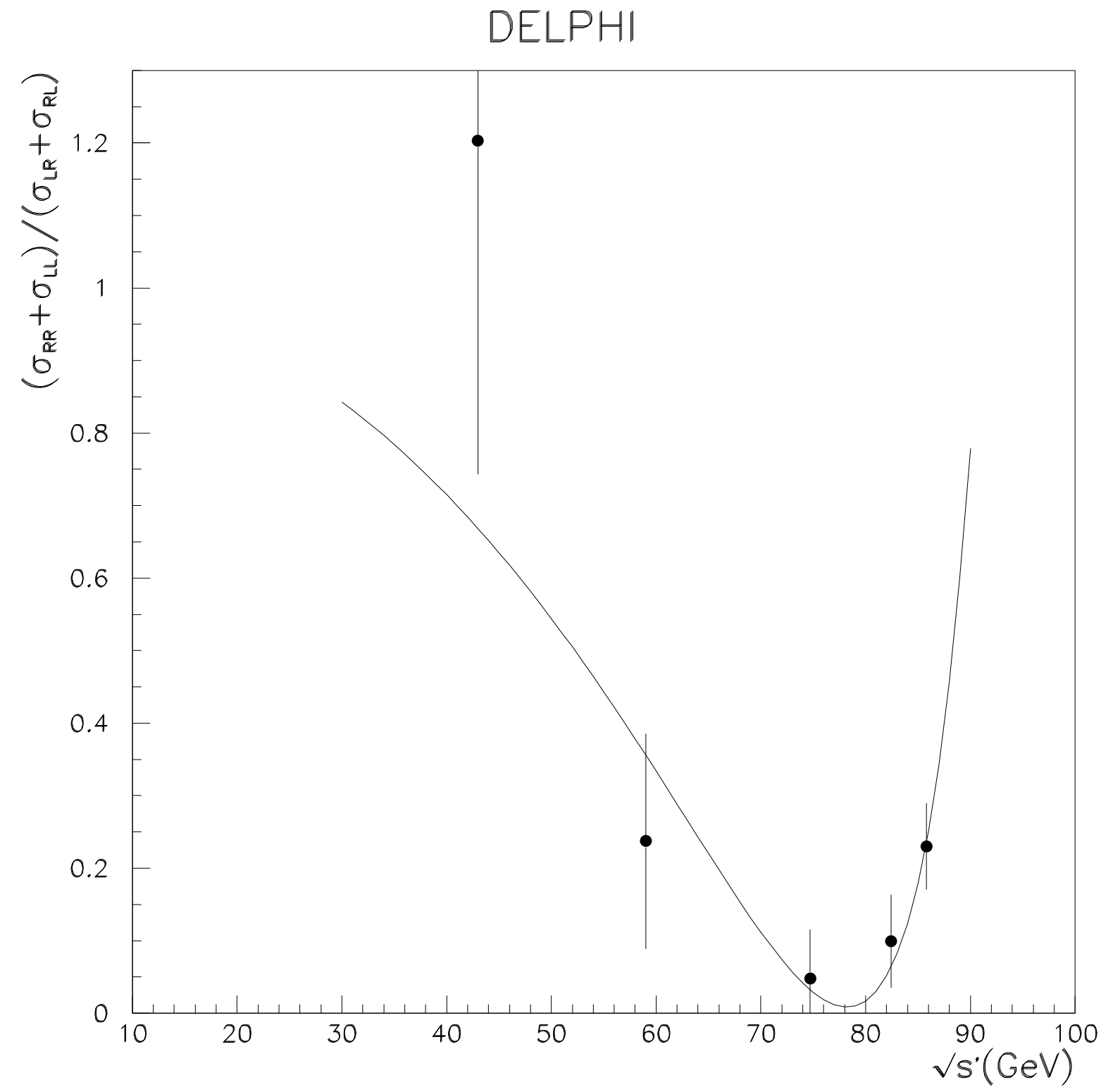

Figure 7: Ratio $\tilde{\sigma}_{+}^{\mu} / \tilde{\sigma}_{-}^{\mu}=\left(\sigma_{R R}+\sigma_{L L}\right) /\left(\sigma_{L R}+\sigma_{R L}\right)$ as a function of the effective annihilation energy. The solid line shows the SM prediction. 